\title{
Cx43 deficiency confers EMT-mediated tamoxifen resistance to breast cancer via c-Src/PI3K/Akt pathway
}

\author{
Deng-Pan $\mathrm{Wu}^{1,2^{*}}$, Yan Zhou ${ }^{*}$, Li-Xiang Hou ${ }^{*}$, Xiao-Xiao Zhu ${ }^{1}$, Wen Yi ${ }^{1}$, Si-Man Yang ${ }^{3}$, Tian-Yu Lin ${ }^{1}$, \\ Jin-Lan Huang ${ }^{1,2}$, Bei Zhang 5 , Xiao-Xing Yin ${ }^{1 凶}$ \\ 1. Jiangsu Key Laboratory of New Drug Research and Clinical Pharmacy, Pharmacy School of Xuzhou Medical University, Xuzhou City, Jiangsu Province, \\ 221004, P.R. China. \\ 2. Department of Pharmacology, Pharmacy School of Xuzhou Medical University, 221004, Xuzhou City, Jiangsu Province, P.R. China. \\ 3. Scientific research center of traditional Chinese medicine, Guangxi University of Chinese Medicine, Nanning City, Guangxi Zhuang Autonomous Region, \\ P.R. China. \\ 4. Clinical Pharmacy, Jingjiang People's Hospital, 214500, Jingjiang City, Jiangsu Province, P.R. China. \\ 5. Department of gynaecology and obstetrics, Xuzhou Central Hospital, 221009, Xuzhou City, Jiangsu Province, P.R. China. \\ *These authors contributed equally to this work.
}

$\square$ Corresponding author: Xiao-Xing Yin, PhD. Jiangsu Key Laboratory of New Drug Research and Clinical Pharmacy, Pharmacy School of Xuzhou Medical University; No.209 Tongshan Road, Xuzhou City, Jiangsu Province, 221004, P.R. China. E-mail: yinxx@xzhmu.edu.cn; Tel: +86 51683262134.

(c) The author(s). This is an open access article distributed under the terms of the Creative Commons Attribution License (https://creativecommons.org/licenses/by/4.0/). See http://ivyspring.com/terms for full terms and conditions.

Received: 2020.11.04; Accepted: 2021.03.30; Published: 2021.06.11

\begin{abstract}
Tamoxifen (TAM) resistance has indicated a significant challenge during endocrine therapy for hormone-sensitive breast cancer. Thus, it is significant to elucidate the molecular events endowing TAM resistance to endocrine therapy. In this study, we found that epithelial-mesenchymal transition (EMT) was an important event to confer TAM resistance, and attenuating EMT by elevating connexin (Cx) 43 expression could reverse TAM resistance. Specifically, Cx43 overexpression improved TAM sensitivity, while Cx43 depletion facilitated TAM insensitivity by modulating EMT in T47D TAM-resistant and -sensitive cells, and transplanted xenografts. Importantly, we found a novel reciprocal regulation between Cx43 and c-Src/PI3K/Akt pathway contributing to EMT and TAM resistance in breast cancer. Moreover, we identified that $\mathrm{Cx} 43$ deficiency was significantly correlated with poor relapse-free survival in patients undergoing TAM treatment. Therefore, Cx43 represents a prognostic marker and an attractive target for breast cancer treatments. Therapeutic strategies designed to increase or maintain $\mathrm{Cx} 43$ function may be beneficial to overcome TAM resistance.
\end{abstract}

Key words: Connexin 43, tamoxifen resistance, epithelial-mesenchymal transition, c-Src/PI3K/Akt pathway

\section{Introduction}

Breast cancer is one of the most common cancer and the second leading causes of cancer-related deaths in women worldwide. Approximately $70 \%-75 \%$ of breast cancer are classified as estrogen receptor-positive $\left(\mathrm{ER}^{+}\right)$breast cancer [1]. For $\mathrm{ER}^{+}$ breast cancer, primary therapy is endocrine treatment. There are reports showing that antiestrogen treatment can dramatically improve the treatment outcome and reduce the recurrence risk for patients with $\mathrm{ER}^{+}$breast cancer [2]. Tamoxifen (TAM) is the most widely used nonsteroidal and antiestrogen agent for $\mathrm{ERa}^{+}$patients [3]. Although initial responses to TAM treatment are positive, approximately $30 \%$ of $\mathrm{ER}^{+}$patients have acquired resistance to TAM therapy and eventually develop local recurrence and distant metastases [1]. Therefore, exploring the molecular mechanisms of TAM resistance and investigating novel targets to overcome acquired TAM resistance in breast cancer is urgently needed.

Epithelial-mesenchymal transition (EMT) is a process by which epithelial cells lose their junctions and polarity and gain migratory and invasive phenotypes to transform into mesenchymal stem cells. The features of EMT include changes in cellular morphology, downregulation of the expression of epithelial cell markers such as E-cadherine (E-ca) and upregulation of mesenchymal markers such as $\mathrm{N}$-cadherin (N-ca) and a-SMA [4]. Emerging data 
highlight that EMT in cancer cells is associated with increased tolerance to chemotherapy and radiotherapy [5], while reversing EMT in resistant cancer cells could increase the cytotoxicity of cancer therapeutic agents [6, 7]. Consistent with these findings, EMT features, including increased cell motility and morphological distinctions have been observed in TAM-resistant human breast cancer cells [8]. Inducing EMT phenotype by TGF- $\beta 1$ or MCAM/ CD146 promotes TAM resistance in breast cancer cells $[9,10]$. Thus, reversing EMT has been considered as a beneficial strategy to conquer acquired TAM resistance in breast cancer.

Connexins (Cxs) are a family of transmembrane proteins, which compose gap junction (GJ) channel between neighboring cells. Gap junction intercellular communication (GJIC) is driven by the diffusion of the signaling molecules smaller than $2 \mathrm{kDa}$ [11]. It is generally believed that the tumorigenic phenotype is widely associated with lack of $\mathrm{Cx}$ expression and GJIC, while restoring $\mathrm{Cx}$ expression and GJIC improved the sensitivity of cancer cells to chemotherapeutics and inhibited tumor growth [12]. Cx43 is the most widely studied isoform of Cxs due to its abundant expression in the heart, breast, brain and colorectal tissues [13]. It has been proven that Cx43 and its derived GJIC act as tumor suppressors to inhibit glioma and colorectal cancer growth [14, 15]. Additionally, Cx43 sensitizes malignant cells to platinum-based drugs. For example, increasing the level of $\mathrm{Cx} 43$ could improve oxaliplatin cytotoxicity in colorectal cancer cell lines [16] and enhance the sensitivity of glioma to temozolomide [14]. Additionally, improving Cx43-mediated gap junctional function by a-connexin carboxyl-terminal (ACT1) peptide could increase the activity of lapatinib and TAM [17]. Nevertheless, the underlying mechanisms of $\mathrm{C} \times 43$ in TAM resistance in breast cancer remain elusive.

In this study, we have explored the potential role of Cx43 in TAM resistance in breast cancer and its underlying mechanisms. We demonstrated that Cx43 deficiency conferred TAM resistance and overexpression of $\mathrm{C} \times 43$ reversed TAM resistance by modulating EMT. Importantly, we found that there was a mutual regulation between $\mathrm{C} \times 43$ and c-Src/ PI3K/Akt pathway contributing to EMT and TAM resistance. Additionally, by analyzing the association of Cx43 expression with the outcome of TAM treatment, we observed that $\mathrm{C} \times 43$ deficiency predicts poor clinical outcome to TAM therapy. This study indicates that $\mathrm{C} \times 43$ could serve as a critical regulator in TAM resistance. Therapeutic strategies designed to elevate or sustain Cx43 function may be beneficial to overcome TAM resistance.

\section{Methods}

\section{Materials}

TAM and 18a-GA were provided by Sigma-Aldrich (St. Louis, MO, USA). TGF- $\beta 1$ was obtained from Pepro Tech (Rocky Hill, NJ, USA). MS-275 was supplied by MedChemExpress (Monmouth Junction, NJ, USA). Antibodies against Cx43 and a-SMA were obtained from Abcam (Cambridge Science Park, Cambridge, England). E-ca, $\mathrm{N}$-ca, p-Akt, Akt, c-Src and p-c-Src antibodies and LY294002 (PI3K inhibitor) were purchased from Cell Signaling Technology (Danvers, MA, USA). $\beta$-actin and GAPDH antibodies were purchased from Bioworld Technology, Co, Ltd. (Nanjing, China). Dasatinib was from Aladdin Bio-Chem Technology Co.LTD (Shanghai, China). IGF-1 was from PeproTech, Inc. (Rocky Hill, USA). Other reagents were all provided from Sigma-Aldrich unless otherwise noted.

\section{Cell culture and treatment}

Mycoplasma-free T47D cells were obtained from the American Type Culture Collection (Manassas, VA, USA). Cells were cultured in RPMI1640 (Gibco, Grand Island, NY, USA) containing 10\% fetal bovine serum (Gibco, Grand Island, NY, USA) and 1\% penicillin/ streptomycin at $37{ }^{\circ} \mathrm{C}$ in water-saturated $5 \% \mathrm{CO}_{2}$ atmosphere. TAM-sensitive T47D (T47D/TS) cells were exposed to increasing concentrations of TAM and maintained in $10^{-6} \mathrm{M}$ TAM for at least 18 months to establish acquired TAM-resistant T47D (T47D/TR) cells. Cells were incubated with $25 \mu \mathrm{mol} / \mathrm{L}$ LY294002 or $2 \mu \mathrm{mol} / \mathrm{L}$ dasatinib for $24 \mathrm{~h}$ to retard the activity of PI3K/Akt signaling or c-Src, respectively. Additionally, cells were treated with IGF-1 (100 $\mathrm{ng} / \mathrm{mL}$ ) for the activation of PI3K/Akt signaling.

\section{Cx43 interference and overexpression}

The lentiviral pGLV3/H1/GFP+Puro vector (GenePharma, China) containing shRNA against $\mathrm{C} \times 43$ and $\mathrm{pCDH} / \mathrm{CMV} / \mathrm{MCS} / \mathrm{EF} 1 / \mathrm{coRFP}+\mathrm{Puro}$ vector (GenePharma, China) containing chimeric $\mathrm{C} \times 43$ was constructed as previously reported [18]. shRNA with no target gene (scramble) was considered as a control. Lentivirus stably transfected to T47D/TS and T47D/TR cells in the presence of $5 \mu \mathrm{g} / \mathrm{mL}$ polybrene (GenePharma, China). After 2 weeks, single independent clones were randomly isolated and plated separately to analyze for Cx43 knockdown or overexpression by western blotting.

\section{Western blotting}

Western blotting protocol was according to previous studies $[19,20]$. Briefly, the extracted protein 
samples were separated and transferred to a nitrocellulose transfer membrane (Excell Bio, China). Primary antibodies (dilution ranging from 1:2000 to 1:5000) were incubated at $4{ }^{\circ} \mathrm{C}$ overnight. Secondary antibody was IRDye 800CW purified immunoglobulin-conjugated anti-rabbit (dilution 1:10000). Immunopositive bands were visualized at $\mathrm{Ex} / \mathrm{Em}=778 \mathrm{~nm} / 795 \mathrm{~nm}$.

\section{CCK-8 assay}

TAM cytotoxicity was assessed by a CCK-8 assay (Dojindo Molecular Technologies, Japan). As previously reported [21], after incubation with different concentrations of TAM for 48 h, $10 \mu \mathrm{L}$ CCK-8 solution was added and incubated with cells for $2 \mathrm{~h}$. The OD values were detected at $450 \mathrm{~nm}$ to assess cellular survival using Enzyme-labeling instrument (Elx808, Bio Tek, America).

\section{"Parachute" dye-coupling assay}

GIIC function was evaluated by "Parachute" dye-coupling assay was performed as described in our previous study [22]. Donor and receiver cells were grown to confluence. Donor cells were loaded with 5 $\mu \mathrm{M}$ calcein-acetoxymethyl ester, which is converted into the gap junction-permeable dye calcein and diffuse through GJ channel. The donor cells were then seeded onto the receiver cells at 1:150 donor/receiver ratio after trypsinization. The donor cells were allowed to contact with the monolayer of receiver cells for GJ formation for $4 \mathrm{~h}$ at $37^{\circ} \mathrm{C}$. The average amount of the receiver cells receiving dyes per donor cell was determined using a fluorescence microscope and normalized to the controls.

\section{Cell invasion assay}

Cell invasion assay was performed to assess the invasive ability as described in a previous study [23]. Briefly, the invaded cells were counted in eight high-power fields of cells in each well under an inverted microscope. The relative invasive rate was determined by the invasive rate of the treated group (number of invaded cells per total cell number) divided by that of the control group.

\section{In vivo xenograft studies}

BALB/c-nude mice (16-22 g, female) were subcutaneously inoculated under the right shoulder with $5 \times 10^{7}$ cells/flank. One week before injection, the mice transplanted with Cx43-deficient and its scramble T47D/TS cells were pretreated with drinking water containing $1 \mu \mathrm{M} \beta$-estradiol (SigmaAldrich). The mice transplanted with Cx43overexpressed and control T47D/TR cells were provided with drinking water supplemented with 1 $\mu \mathrm{M} \beta$-estradiol (Sigma-Aldrich) and pasted with estradiol sustained-release patch (1.25 mg per mice) (Zhejiang Yatai Pharmaceutical Co., Ltd., Zhejiang, China). When the volume of tumors reached to 100 $\mathrm{mm}^{3}$, the mice were intragastrically administrated with vehicle (control) or $30 \mathrm{mg} / \mathrm{kg}$ TAM for 10 days. Tumor dimensions were measured by a caliper every 2 days. The tumor volume (V) was calculated as $\left(\right.$ length $\times$ width $\left.^{2}\right) \times 0.5$. The relative tumor volume (RTV) of each tumor was defined as the ratio of the volume at a given time and the volume prior to treatment [24]. On day 10, after their tumor sizes were measured, all mice were sacrificed and the xenografts were excised and weighted. Meanwhile, the expression of EMT biomarkers of xenografts were detected. All animal experiments were approved by the Guidance Suggestions for Caring for Laboratory Animals issued by the Ministry of Science and Technology of China in 2006.

\section{Immunohistochemistry (IHC) for tissue microarray}

Commercial breast cancer tissue microarray (TMA) slides (BC081120e and HBreD140Su05) were purchased from US Biomax, Inc. (Maryland, USA) and Shanghai Outdo Biotech Co. Ltd. (Shanghai, China), respectively. All tissues were collected with the patients consenting to the use of their tissues and clinical data. IHC staining against $\mathrm{C} \times 43, \mathrm{E}-\mathrm{ca}$ and a-SMA was carried out with EliVision ${ }^{\mathrm{TM}}$ plus kit (Maxim biotechnology co. Ltd, Fuzhou, China). The staining was evaluated blindly and independently by two pathologists. The intensity of staining was scored 0 to 3 ( $0=$ negative, $1=$ low, $2=$ moderate, $3=$ high). The percentage of positively stained cells was recorded and divided into four categories: 1 (0\%-25\%), 2 $(26 \%-50 \%), 3(51 \%-75 \%)$ and 4 (76\%-100\%). The expression level was calculated by multiplying the scores of staining intensity and the percentage of positive cells [25].

\section{Co-IP assay}

Cell samples were lysed in iced cold PIPA buffer and the supernatants were collected by centrifuging at $14000 \mathrm{rpm}$. The lysates were incubated with Cx43 antibody and protein A/G agarose gel overnight. The gel was washed with RIPA buffer, boiled with SDS loading buffer and subjected to SDS-PAGE resolution. After SDS-PAGE, c-Src antibody was used to detect the interaction of these two proteins by Western blotting assay [26].

\section{Statistical analysis}

The data were expressed as mean $\pm S D$. The variance was analyzed using the SPSS software for Windows 17.0 using one-way analysis of variance 
(ANOVA) or $t$-test. $P<0.05$ was considered to be statistically significant.

\section{Results}

Cx43 expression significantly correlates with clinicopathological parameters and clinical outcome to TAM therapy

For investigating the role of $\mathrm{Cx} 43$ expression in breast cancer, we interrogated the Cancer Genome Atlas (TCGA) database and found that the expression of Cx43 was significantly decreased in primary breast cancer and its subtypes (1097 samples) than that in normal breast samples (114 samples) (Fig. 1A). We then examined the correlation of $\mathrm{Cx} 43$ expression with clinicopathological parameters using immunohistochemistry (IHC) in 250 breast cancer samples (tissue microarray). The results showed that

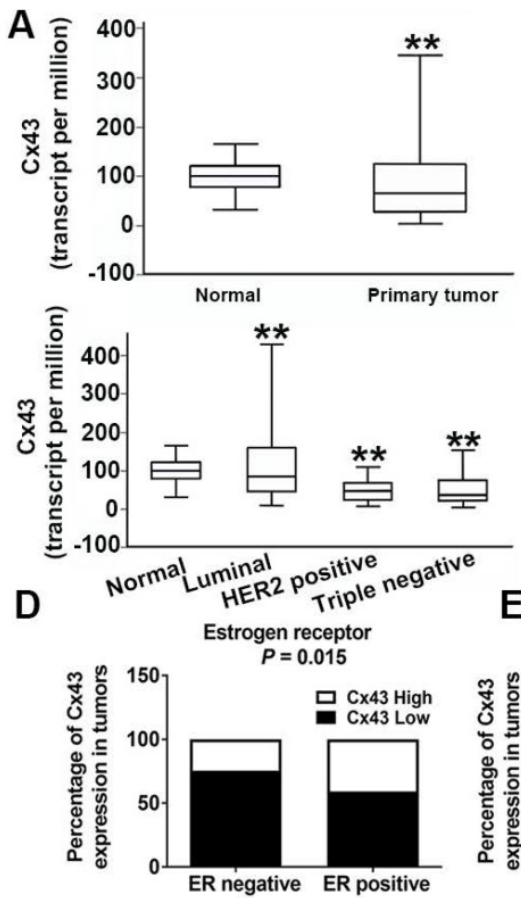

B
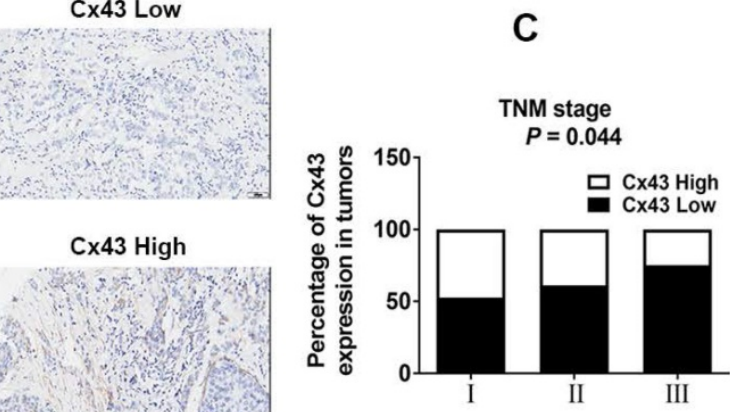

G
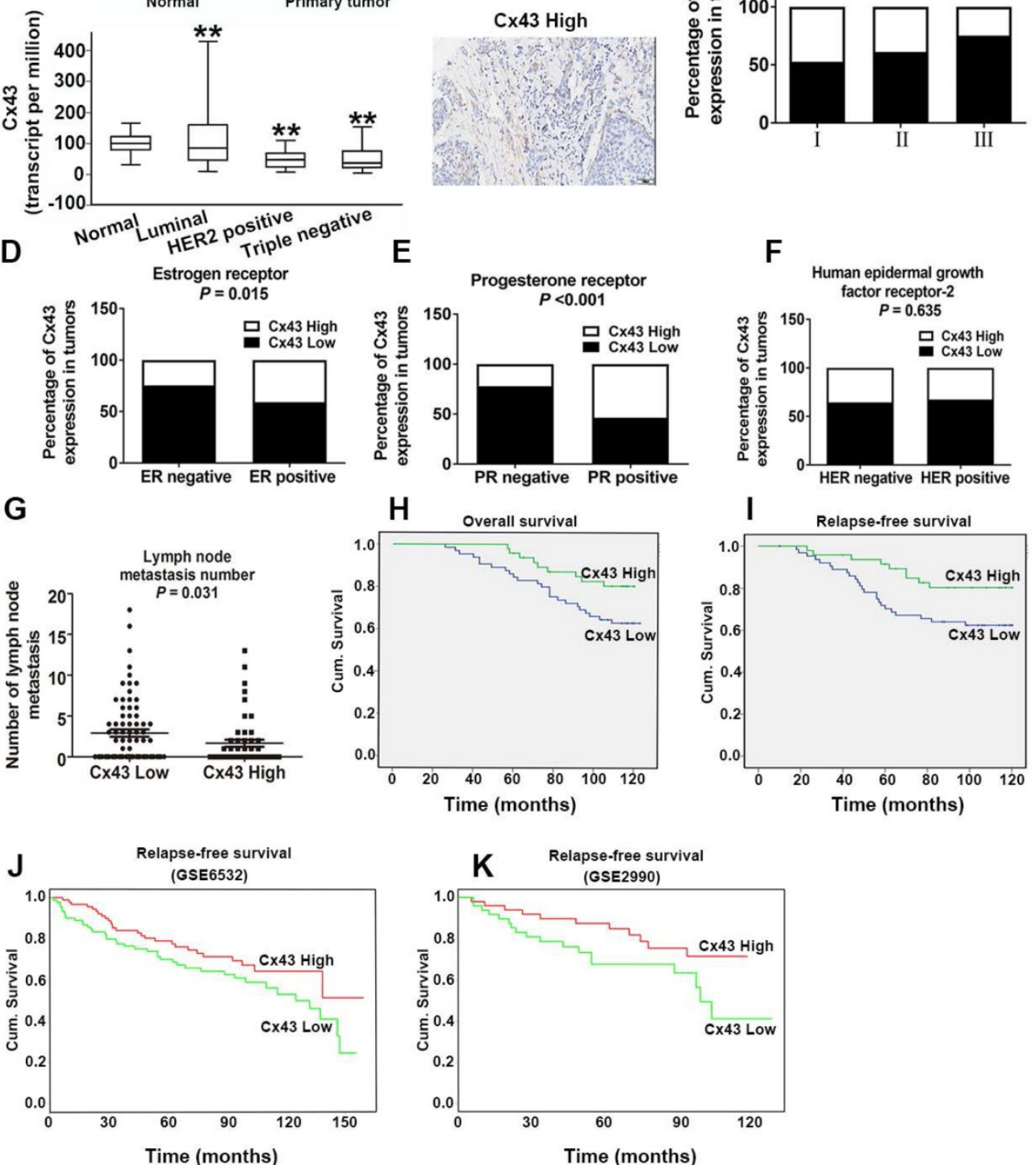

Figure 1. Cx43 expression significantly correlates with clinicopathological parameters and clinical outcomes of TAM therapy. (A) Cx43 mRNA expression in primary breast cancer and its subtypes from TCGA database. $* * P<0.01$ versus normal breast tissue. (B) Weak and strong Cx43 staining in breast cancer tissue. Scale bar, $100 \mu \mathrm{m}$. (C-F) Percentages of human breast cancer samples with high level of $\mathrm{Cx} 43$ expression in different tumor subtypes and tumor grades ( $X^{2}$ test). (G) Correlation of $\mathrm{Cx} 43$ expression with lymph node metastasis number (unpaired $t$-test). $(\mathrm{H}, \mathrm{I})$ High $\mathrm{Cx} 43$ expression correlated with a higher 10-year overall survival and relapse-free survival for 127 breast cancer patients $(P<0.05$, log-rank test). (J, K) High Cx43 expression correlated with a higher 10-year relapse-free survival for breast cancer patients from GEO datasets $(P<0.05$, log-rank test). 
low Cx43 expression was correlated with high TNM stage (Fig. 1C). Moreover, low Cx43 expression was markedly correlated with ER and PR negative, but not significantly correlated with HER-2 expression (Fig. 1D-F). Interestingly, low Cx43 level was significantly correlated with high lymph node metastasis (Fig. 1G). Furthermore, the results of Kaplan-Meier survival analysis indicated that low Cx43 expression was correlated with low 10-year overall survival and relapse-free survival (Fig. $1 \mathrm{H}$ and I). The relationship between Cx43 expression and survival analysis was further analyzed by Kaplan-Meier estimates based on
KM plotter online database (www.kmplot.com) containing 3951 breast cancer samples. The results revealed that low $\mathrm{C} \times 43$ expression was correlated with a poor outcome in overall survival and relapse-free survival (Fig. 2A and B). Furthermore, patients with lower expression of $\mathrm{C} \times 43$ had greater decreased relapse-free survival in luminal A subtype of breast cancer (Fig. 2C). In addition, in $\mathrm{ER}^{+}$but not

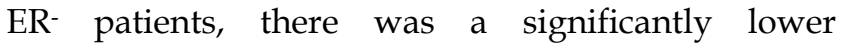
probability of survival for patients with lower Cx43 expression (Fig. 2E and F).
A
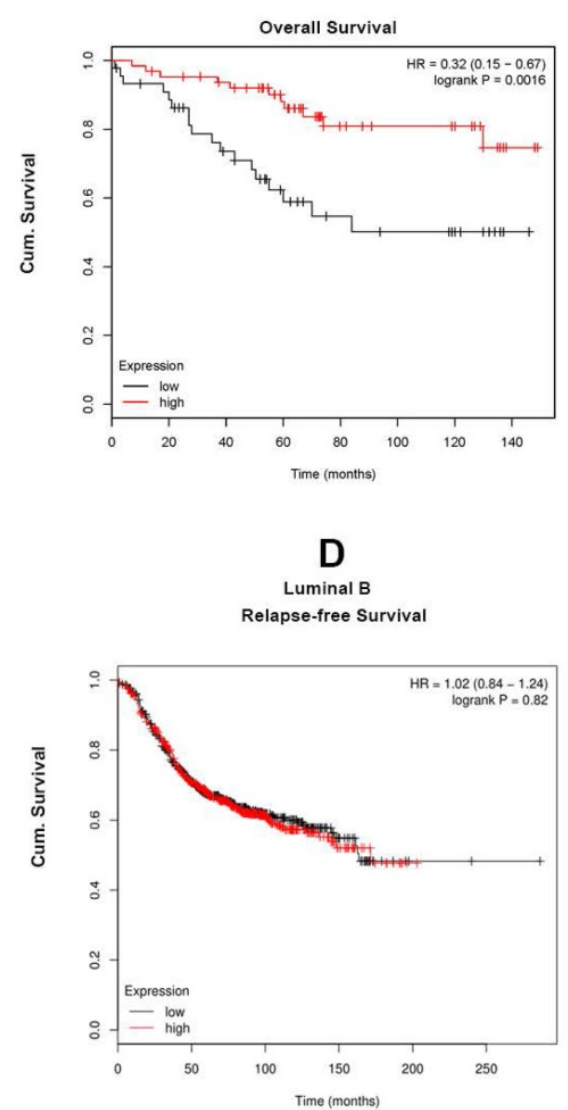

G

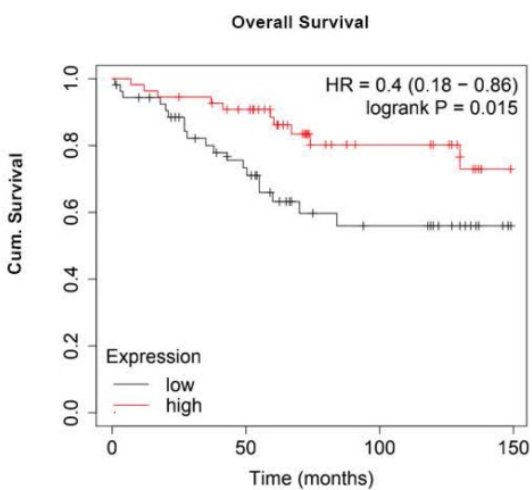

B

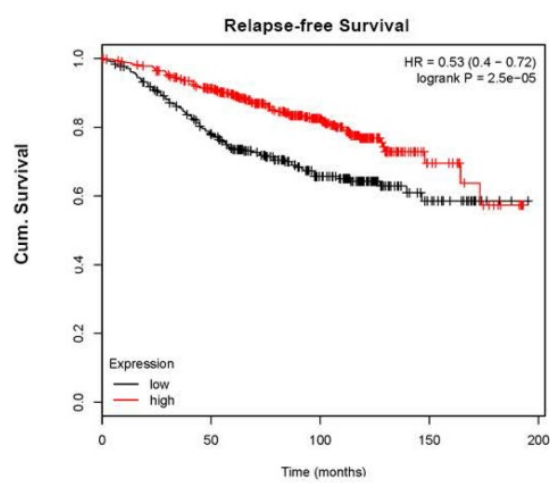

E

ER positive

Relapse-free Survival

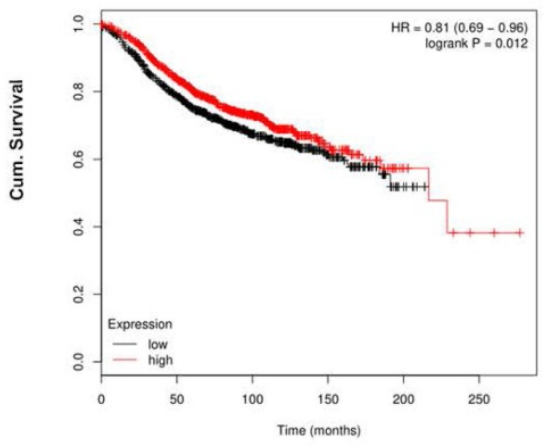

H

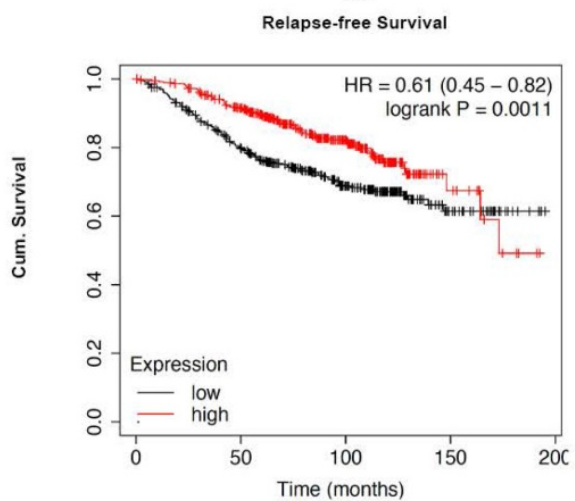

Figure 2. The relationship between Cx43 expression and survival analysis in the subtypes of breast cancer. The correlation of Cx43 expression with survival analysis was analyzed by Kaplan-Meier estimates based on KM plotter online database (www.kmplot.com). 
We next performed a meta-analysis based on TCGA (809 samples) datasets to investigate whether Cx43 expression prognosticates TAM resistance. The results showed that low $\mathrm{C} \times 43$ expression in breast cancer was significantly correlated with poor outcome in overall survival and relapse-free survival with restriction of TAM treatment (Fig. $2 \mathrm{G}$ and $\mathrm{H}$ ). We further analyzed the GEO datasets (GSE6532 and GSE2990) of which the patients administrated with TAM monotherapy using Kaplan-Meier survival analysis after each dataset was defined into groups with high and low Cx43 expression [27]. The results revealed that the cohorts expressing low level of $\mathrm{Cx} 43$ displayed a higher possibility of developing recurrence as compared to the cohorts expressing high level of $\mathrm{Cx} 43$ (Fig. 1J and K). The data imply that Cx43 deficiency predicts TAM resistance.

Collectively, these results from both our clinical specimens and database indicate that $\mathrm{C} \times 43$ expression serves as a predictor of clinical outcome to TAM therapy.

\section{Acquired TAM-resistant breast cancer cells exhibited EMT characteristics and Cx43 deficiency}

It has been established that ER ${ }^{+}$MCF-7 and BT-474 cell lines expressed low level of Cx43, whereas relatively high level of $\mathrm{Cx} 43$ was detected in $\mathrm{ER}^{+} \mathrm{T} 47 \mathrm{D}$ cells $[28,29]$. Therefore, in order to investigate the role of Cx43 in TAM resistance, T47D cell line was selected to establish acquired TAM-resistant cells (T47D/TR) by continuous exposure to stepwise increasing concentrations of TAM and maintained in $10^{-6} \mathrm{M}$ TAM for at least 18 months. As shown in Fig. 3A, T47D/TR cells exhibited less sensitivity to TAM than their parental cells with IC50 of $4.249 \times 10^{-4} \mathrm{M}$ versus $7.02 \times 10^{-5} \mathrm{M}$, respectively. Moreover, T47D/TR cells showed an elongated, scattered and mesenchymallike morphology, while their parental T47D cells exhibited tight cell-cell junctions and typical epithelial cobblestone appearance (Fig. 3B). Besides, the expression of E-ca, an epithelial marker, was significantly decreased, whereas the level of mesenchymal markers $\mathrm{N}-\mathrm{ca}$ and a-SMA were substantially elevated (Fig. 3C-E). The increased invasive capability is considered as an important feature of malignant cells undergoing EMT. As illustrated in Fig. 3G, the invasive potential of T47D/TR cells was greatly enhanced compared with their parental cells. These results suggest a potential role of EMT in acquired TAM resistance of breast cancer cells. Additionally, the expression level of Cx43 was significantly reduced in T47D/TR cells (Fig. 3F), indicating that $\mathrm{C} \times 43$ may play a role in reversing EMT and acquired TAM resistance in breast cancer.

\section{EMT switch modulates Cx43 expression and TAM sensitivity}

The finding that TAM resistant cells exhibited the EMT phenotype suggests that EMT switch may affect TAM sensitivity. Studies showed that TGF- $\beta 1$ could induce EMT progression via Smad signaling pathway in different types of malignant cells [4, 10]. To explore the role of EMT in TAM susceptibility, after T47D/TS cells were treated with TGF- $\beta 1$, the expression of EMT markers were detected and the cytotoxicity of TAM was measured. As shown in Fig. $4 \mathrm{~A}$ and B, TGF- $\beta 1$ repressed E-ca expression, induced $\mathrm{N}-\mathrm{ca}$ and a-SMA expression and promoted the invasion of T47D/TS cells, indicating that EMT programme was initiated. Moreover, TGF- $\beta 1$ treatment resulted in a significant downregulation of Cx43 expression and a substantial decrease in TAM cytotoxicity (Fig. 4A and C). The results suggest that EMT induction by TGF- $\beta 1$ prohibits $C x 43$ expression and leads to a decrease in TAM sensitivity.

It has been reported that Entinostat (MS-275), a histone deacetylase (HDAC) inhibitor, could trigger the conversion of EMT into mesenchymal-epithelial transition (MET), and thus inhibiting EMT process $[30,31]$. To explore the role of EMT repression in TAM resistance, after T47D/TR cells were treated with MS-275, the expression of EMT markers and TAM cytotoxicity were measured. As shown in Fig 4D and G, MS-275 induced E-ca expression, suppressed N-ca and a-SMA expression and inhibited the invasion of T47D/TR cells, indicating that MET, a reverse process of EMT, was activated. Additionally, MS-275 treated cells led to the upregulation of Cx43 and exhibited substantially increased sensitivity of T47D/TR cells to TAM (Fig. 4E and F). These results indicate that inhibition of EMT in T47D/TR cells promotes Cx43 expression and reverses TAM resistance.

Collectively, the above results demonstrate that EMT switch modulates Cx43 expression and TAM susceptibility in breast cancer.

\section{Cx43 reverses TAM sensitivity by attenuating EMT in TAM-sensitive cells in a GJIC-dependent manner}

The findings that EMT modulates Cx43 expression and TAM sensitivity prompt us to bring about a scenario that $\mathrm{C} \times 43$ might play a role in modulating EMT and TAM sensibility. We firstly examined the correlation of Cx43 expression and EMT marker E-ca and a-SMA expressions in clinical specimens using IHC. The results showed that high Cx43 expression was positively correlated with E-ca expression, but negatively correlated with a-SMA expression (Fig. 5A and B), indicating that $\mathrm{C} \times 43$ may be implicated in regulating EMT process. 
A

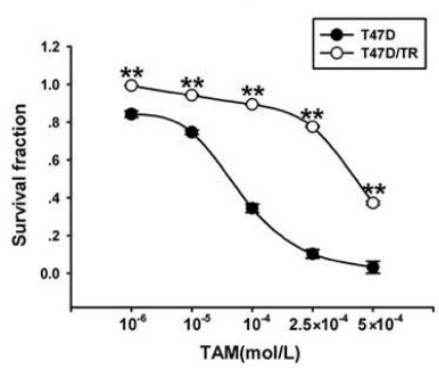

C
B
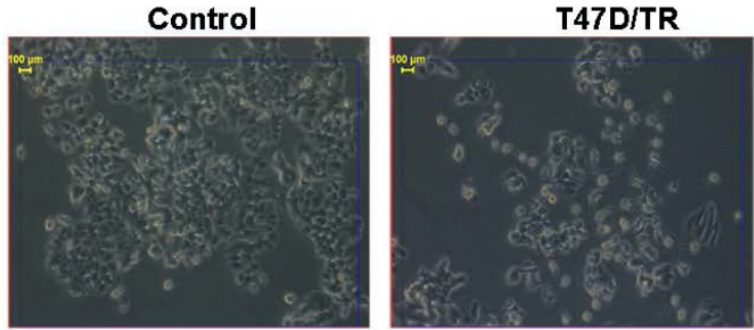
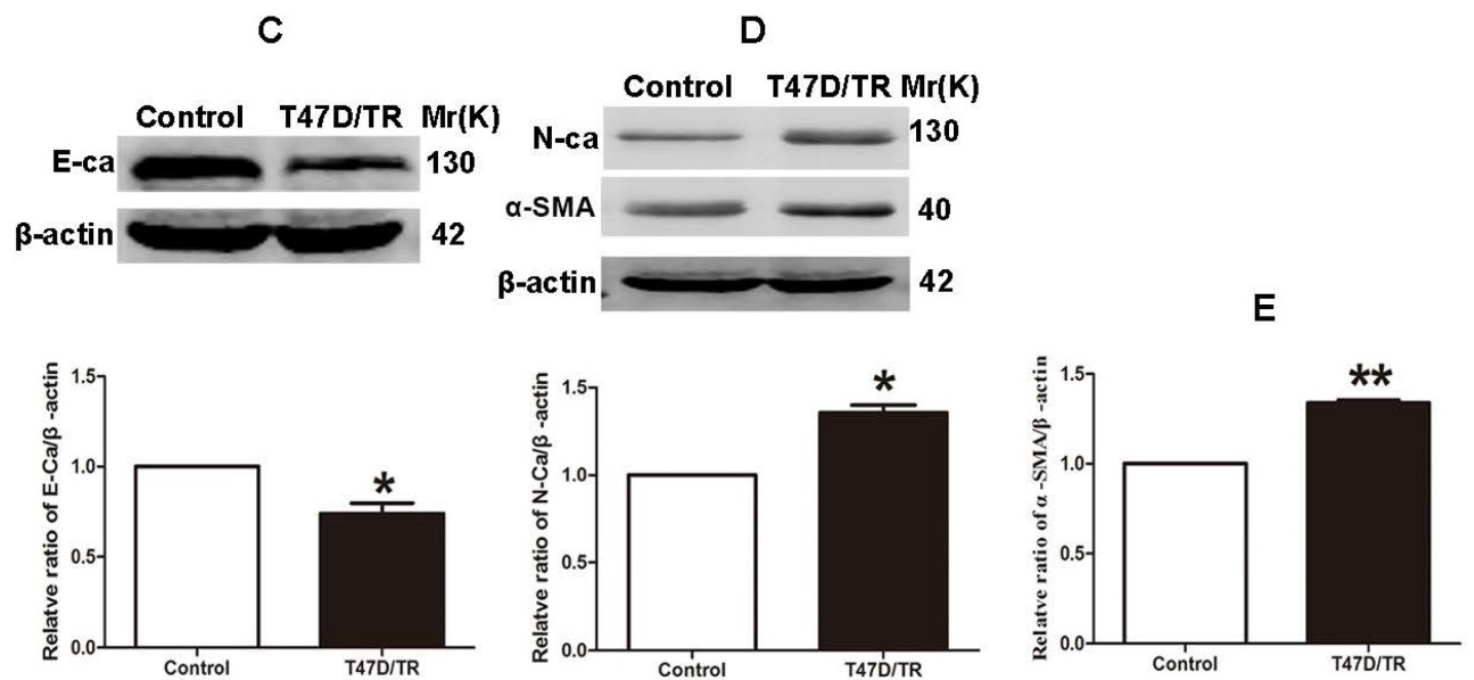

G
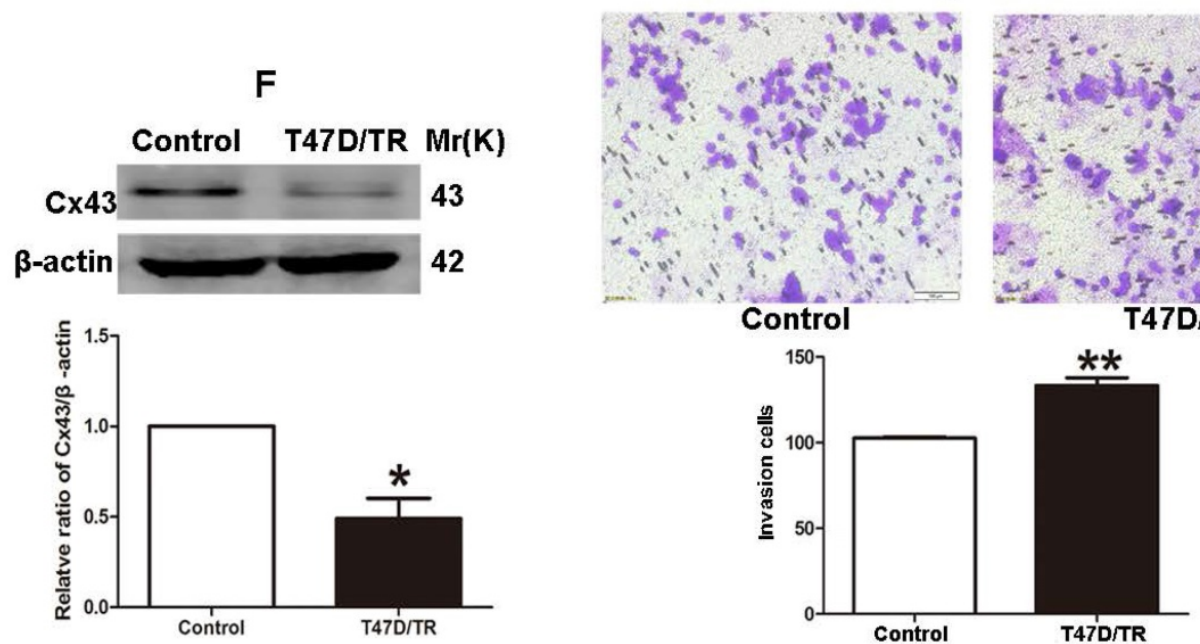

Figure 3. Acquired TAM-resistant breast cancer cells exhibited EMT phenotypes and decreased Cx43 expression. (A) Survival fractions of T47D/TR and parental cells treated with TAM were detected by CCK-8 assay. Data are mean \pm SD from 4 independent experiments. $* * P<0.01$ versus parental cells. (B) Morphological changes of T47D/TR cells and parental cells. Original magnification, $\times 400$. (C-F) The expressions of E-ca, N-ca, $\alpha$-SMA, and Cx43 in T47D/TR cells were detected by western blotting assay, respectively. Data are mean $\pm S D$ from 3 independent experiments. $* P<0.05$, $* * P<0.01$ versus control group. (G) The invasive ability of T47D/TR cells was detected by transwell assay. Data are mean $\pm S D$ from 3 independent experiments. $* * P<0.01$ versus control group. Original magnification, $\times 100$.

We then investigated the role of Cx43 in EMT in breast cancer cells. We firstly built Cx43overexpressed T47D/TS cells to perform gain-offunction assay (Fig. 5C). As shown in Fig 5E and F, Cx43 overexpression reversed TGF- $\beta 1$-induced E-ca downregulation, N-ca and a-SMA upregulation and increment of invasive ability, suggesting that overexpression of $\mathrm{Cx} 43$ was sufficient to inhibit
TGF- $\beta 1$-induced EMT activation. To determine the role of Cx43 in TGF- $\beta 1$-incuded TAM resistance, TAM cytotoxicity was measured. As illustrated in Fig 5G, Cx43 overexpression retarded TGF- $\beta 1$-induced decline in TAM cytotoxicity, indicating that Cx43 overexpression represses TGF- $\beta 1$-incuded TAM insensitivity. 

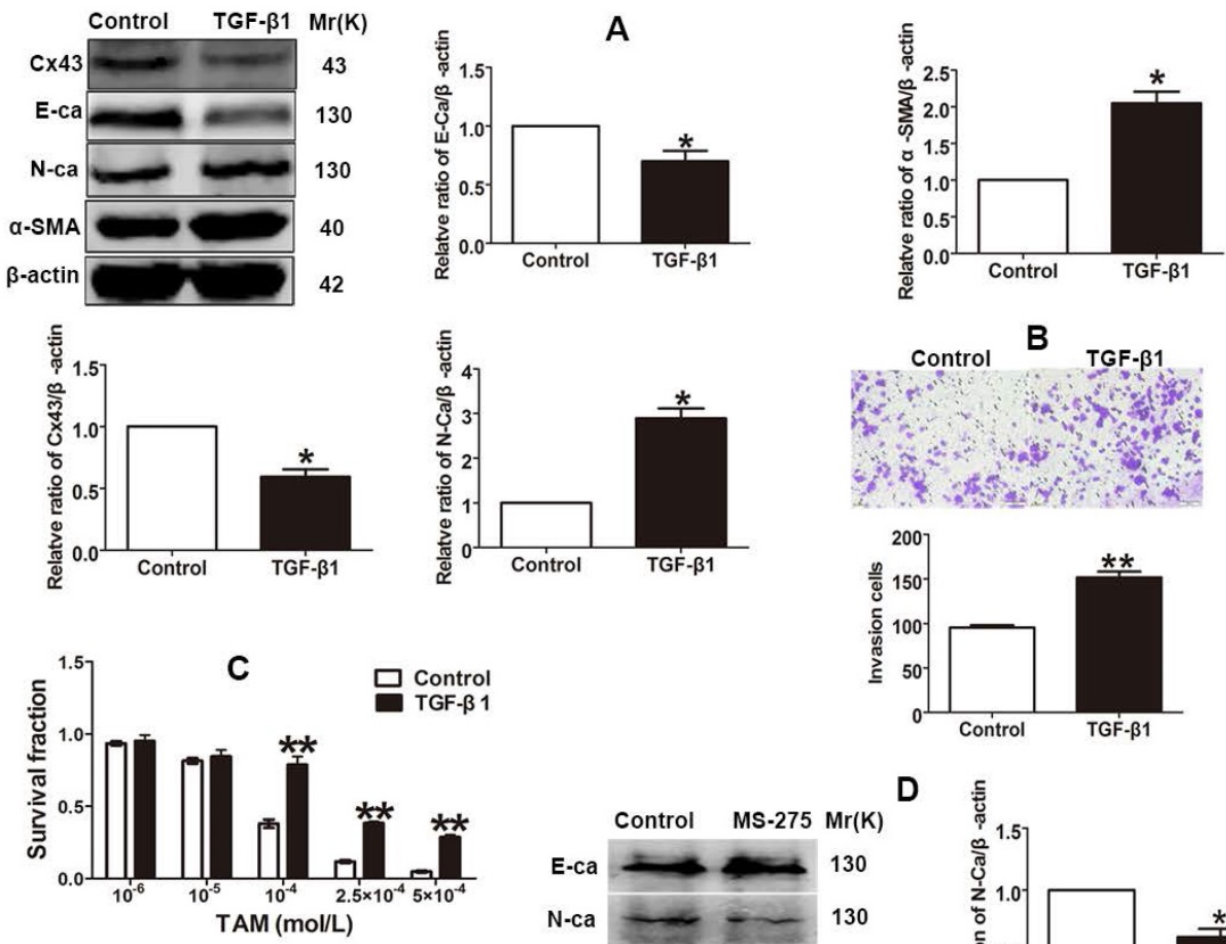

E
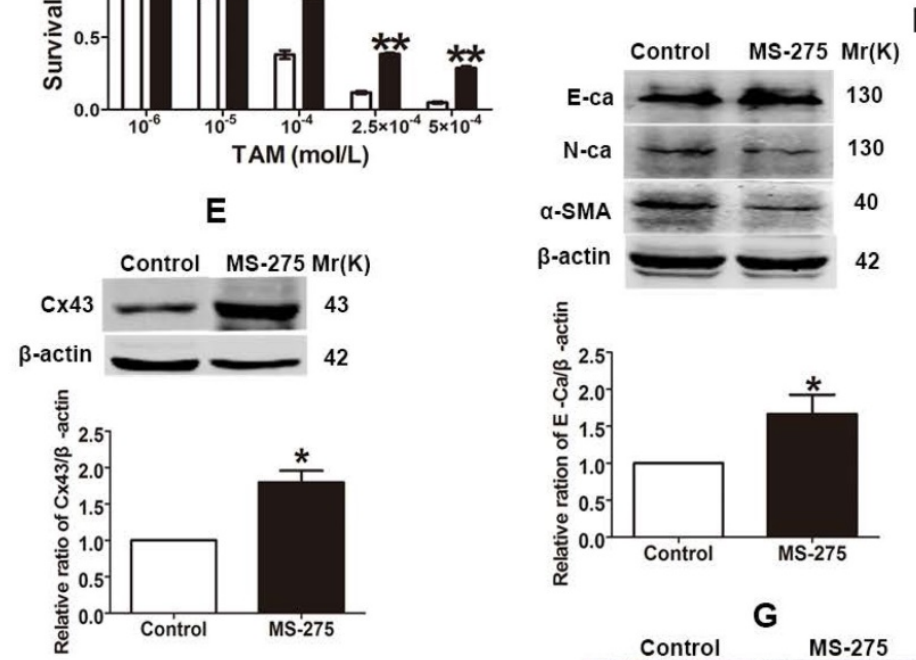

D
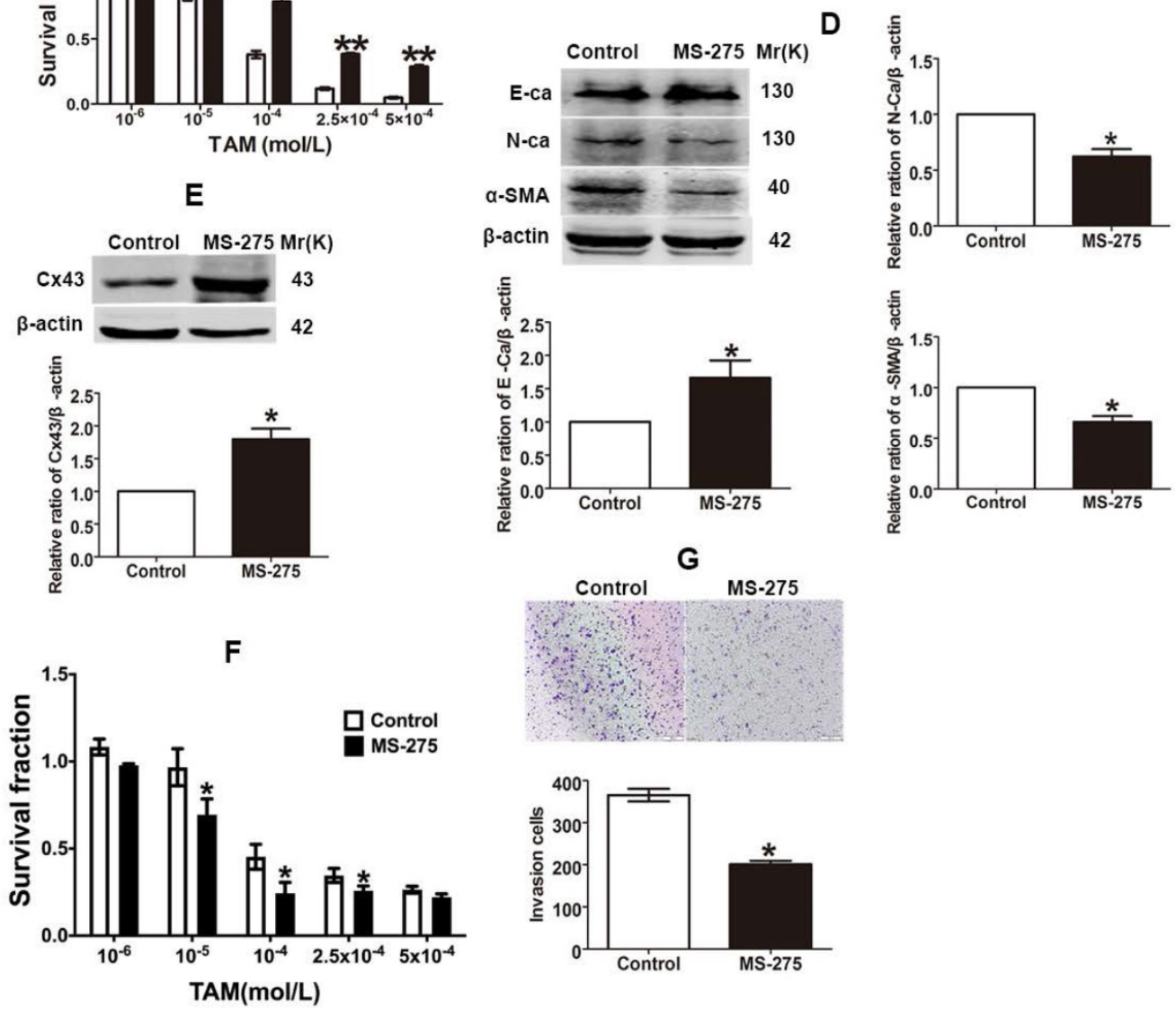

Figure 4. Effects of EMT switch on Cx43 expression and TAM sensitivity in T47D/TS and T47D/TR cells. (A) Effect of TGF- $\beta 1$ on E-ca, N-ca, $\alpha$-SMA and Cx43 expression in T47D/TS cells. (B) Effect of TGF- $\beta 1$ on invasive ability in T47D/TS cells. Original magnification, $\times 100$. (C) Effect of TGF- $\beta 1$ on TAM sensitivity in T47D/TS cells. (D, E) Effect of MS-275 on E-ca, N-ca, a-SMA and Cx43 expression in T47D/TR cells, respectively. (F) Effect of MS-275 on TAM sensitivity in T47D/TR cells. (G) Effect of MS-275 on invasive ability in T47D/TR cells. Original magnification, $\times 100$. Data are mean $\pm S D$ from 3 independent experiments. $* P<0.05, * * P<0.01$ versus control group.

Since GJIC has been reported to regulate cellular differentiation and chemosensitivity $[12,32]$, we then investigated whether Cx43-composed GJIC was involved in the TGF- $\beta 1$-induced EMT and TAM insensitivity. First, parachute assay was performed to assess GJIC after Cx43-overexpressed cells were treated with $10 \mu \mathrm{M}$ 18a-glycyrrhetinic acid (18a-GA), a non-selective GJIC inhibitor [22]. As shown in Fig. 5D, 18a-GA treatment led to reduced GJIC in Cx43-overexpressed T47D/TS cells. Furthermore, we assessed the effect of 18a-GA on EMT and TAM sensitivity in TGF- $\beta 1$-treated Cx43-overexpressed cells. As illustrated in Fig. 5E and F, 18a-GA treatment reversed TGF- $\beta 1$-induced reduction of E-ca 
expression, enhancement of $\mathrm{N}$-ca and a-SMA expression and invasive potential of Cx43overexpressed cells. Moreover, 18a-GA retarded the inhibitory role of $\mathrm{C} \times 43$ in TGF- $\beta 1$-induced TAM insensitivity (Fig. 5G). These results indicate that GJIC may participate in the reversing effect of $\mathrm{C} \times 43$ on TGF- $\beta 1$-incuded EMT and TAM insensitivity.

For further confirming the role of Cx43 in EMT and TAM sensitivity, loss-of-function assay was performed. We transducted two Cx43 short hairpin RNAs (shRNA, shCx43) into T47D/TS cells and found that shRNA1143 had higher efficacy in Cx43 depletion (Fig. 6A). Thus, shRNA1143 was chosen to build Cx43-depleted T47D/TS cell line. As shown in Fig.
6B-D, Cx43-depleted cells exerted significantly enhanced expression of $\mathrm{N}-\mathrm{ca}$ and a-SMA, reduced expression of E-ca, increased invasive ability and decreased TAM sensitivity as compared to the cells transfected with scramble shRNA (control group), suggesting a role of $\mathrm{C} \times 43$ depletion in EMT activation and TAM insensitivity. Furthermore, MS-275 strongly attenuated Cx43-depletion induced EMT and TAM insensitivity.

Taken together, these results indicate that Cx43 attenuates EMT activation and increases TAM sensitivity in TAM-sensitive cells in a GJIC-dependent manner.
A
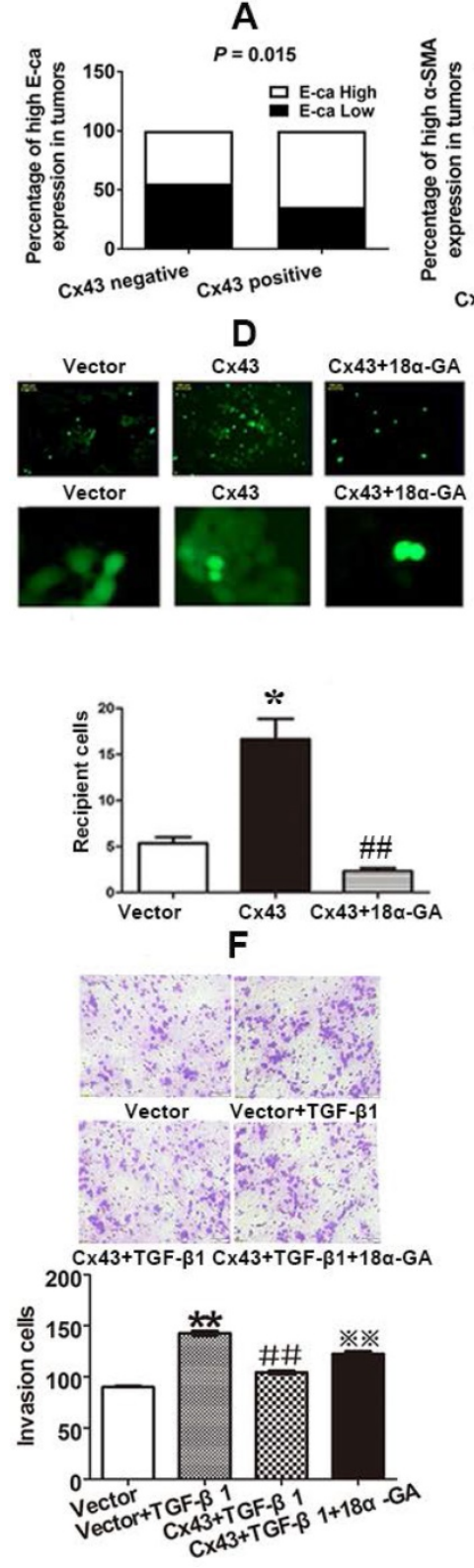

B
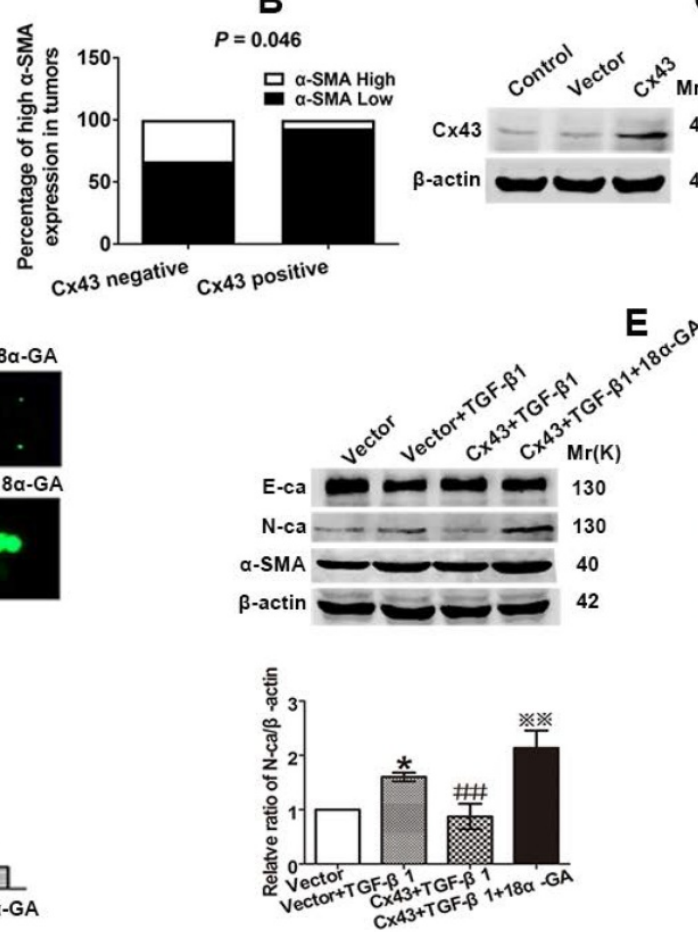

G
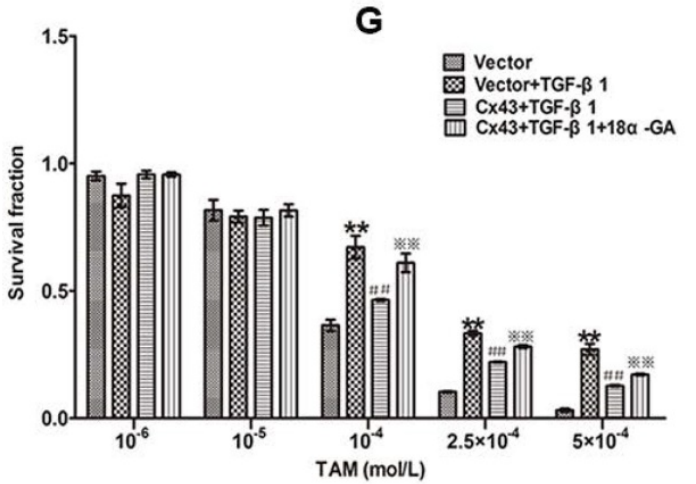

Figure 5. Cx43 suppresses EMT and increases TAM sensitivity in T47D/TS cells in a GJIC-dependent manner. (A, B) Correlation of Cx43 expression with E-ca and $\alpha$-SMA expression ( $X^{2}$ test). (C) Cx43 overexpression efficacy. (D) Effect of 18a-GA on GJIC determined by "Parachute" dye-coupling assay. (E) Effect of Cx43 overexpression on E-ca, $\mathrm{N}$-ca and a-SMA expression, respectively. (F) Effect of Cx43 overexpression on invasive ability. Original magnification, $\times 100$. (G) Effect of Cx43 overexpression on TAM sensitivity. Data are mean \pm SD from 3 independent experiments. $* P<0.05, * * P<0.01$ versus vector group, ${ }^{\prime} P<0.01$, versus vector $+T G F-\beta 1$ group, $* P<0.05$, $* * P<0.01$, versus Cx43+TGF- $\beta 1$ group. Data are mean \pm SD from 3 independent experiments. $* P<0.05$ versus vector group, \#P<0.01, versus $C \times 43$ group. 

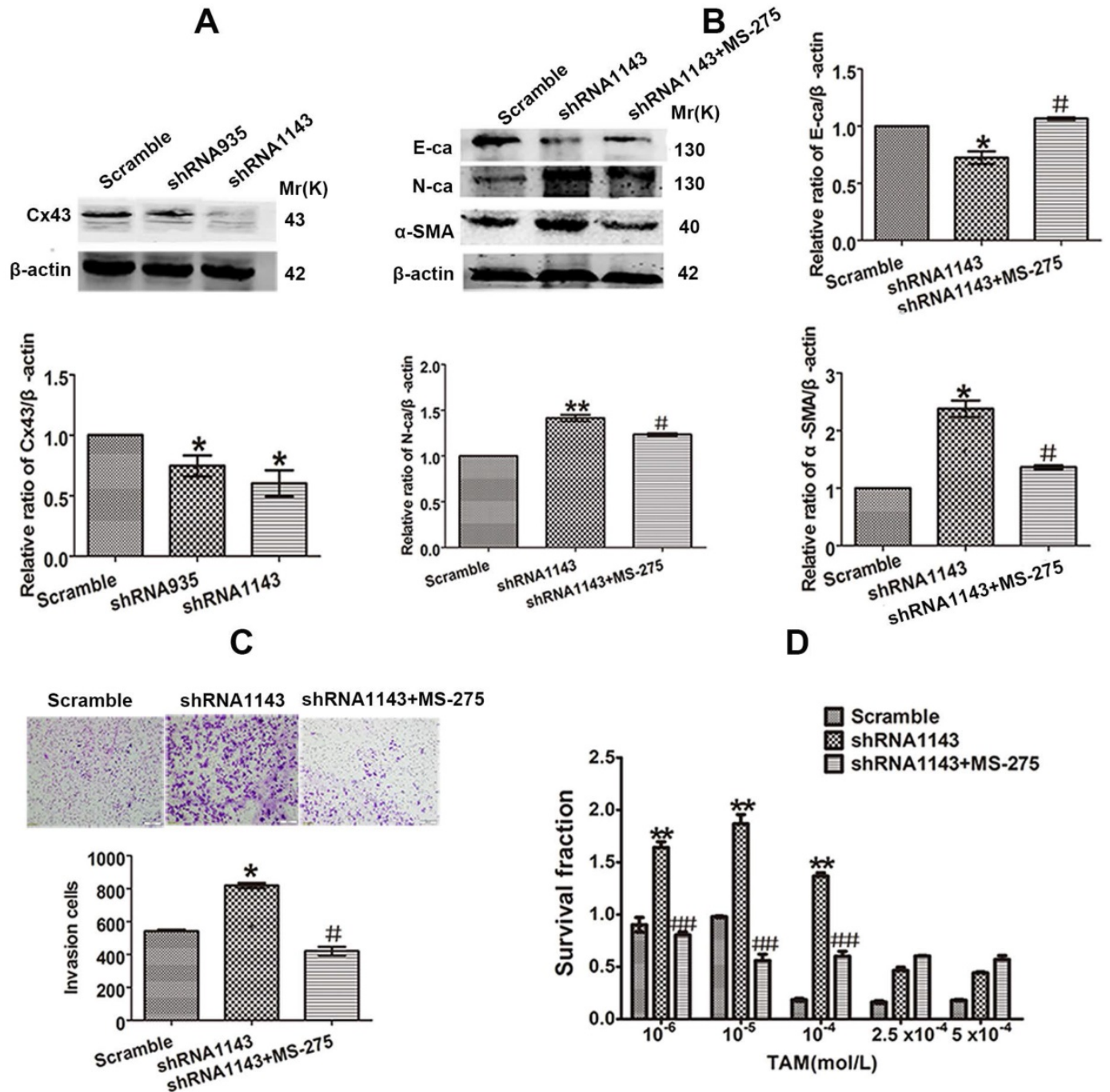

Figure 6. Effect of Cx43 depletion on EMT and TAM sensitivity. (A, B) Effect of Cx43 knockdown on E-ca, N-ca and a-SMA expression. (C) Effect of Cx43 knockdown on invasive ability. Original magnification, $\times 100$. (D) Effect of $C \times 43$ knockdown on TAM sensitivity. Data are mean $\pm S D$ from 3 independent experiments. $* P<0.05$, **P $<0.01$ versus scramble group, $\# P<0.05, \# P<0.01$ versus shRNA1 143 group.

\section{Cx43 suppresses EMT and increases TAM sensitivity in TAM-resistant cells in a GJIC-independent manner}

The inhibitory role of Cx43 in EMT and TAM sensitivity in T47D/TS cells indicates that Cx43 may play a role in reversing TAM resistance. In order to investigate the effect of $\mathrm{Cx} 43$ in EMT and TAM resistance, we established Cx43-overexpressed and -silenced T47D/TR cell lines, and EMT and TAM sensitivity were evaluated in these two cell lines. The results showed that overexpression of $\mathrm{C} \times 43$ significantly enhanced E-ca expression, inhibited N-ca and a-SMA expression and invasive ability of T47D/ TR cells (Fig. 7A-C). Whereas knockdown of Cx43 expression remarkably attenuated E-ca expression, increased N-ca and a-SMA expression and elevated invasive potential of T47D/TR cells (Fig. 7E-G), suggesting that $\mathrm{C} \times 43$ suppresses EMT in acquired TAM-resistant cells. Besides, Cx43 overexpression improved TAM sensitivity, while Cx43 depletion repressed TAM sensitivity in T47D/TR cells (Fig. 7D and $\mathrm{H}$ ). These results indicate that $\mathrm{C} \times 43$ is sufficient to reverse TAM resistance.

To explore the effect of GJIC on Cx43-meidated reversion of EMT and TAM resistance, we assessed the role of 18a-GA in EMT and TAM cytotoxicity in Cx43-overexpressed T47D/TR cells. The results showed that pretreating cells with $10 \mu \mathrm{M} 18 \mathrm{a}-\mathrm{GA}$, a GJIC inhibitor shown to remarkably suppress GJIC at this concentration (Fig. 5D), did not cause a significant difference in EMT and TAM sensitivity (Fig. 7B-D). The results suggest that GJIC was not involved in Cx43-mediated reversion of EMT and TAM resistance in T47D/TR cells.

Collectively, the above results suggest that Cx43 reverses TAM resistance by attenuating EMT in acquired TAM-resistant cells in a GJIC-independent manner. 

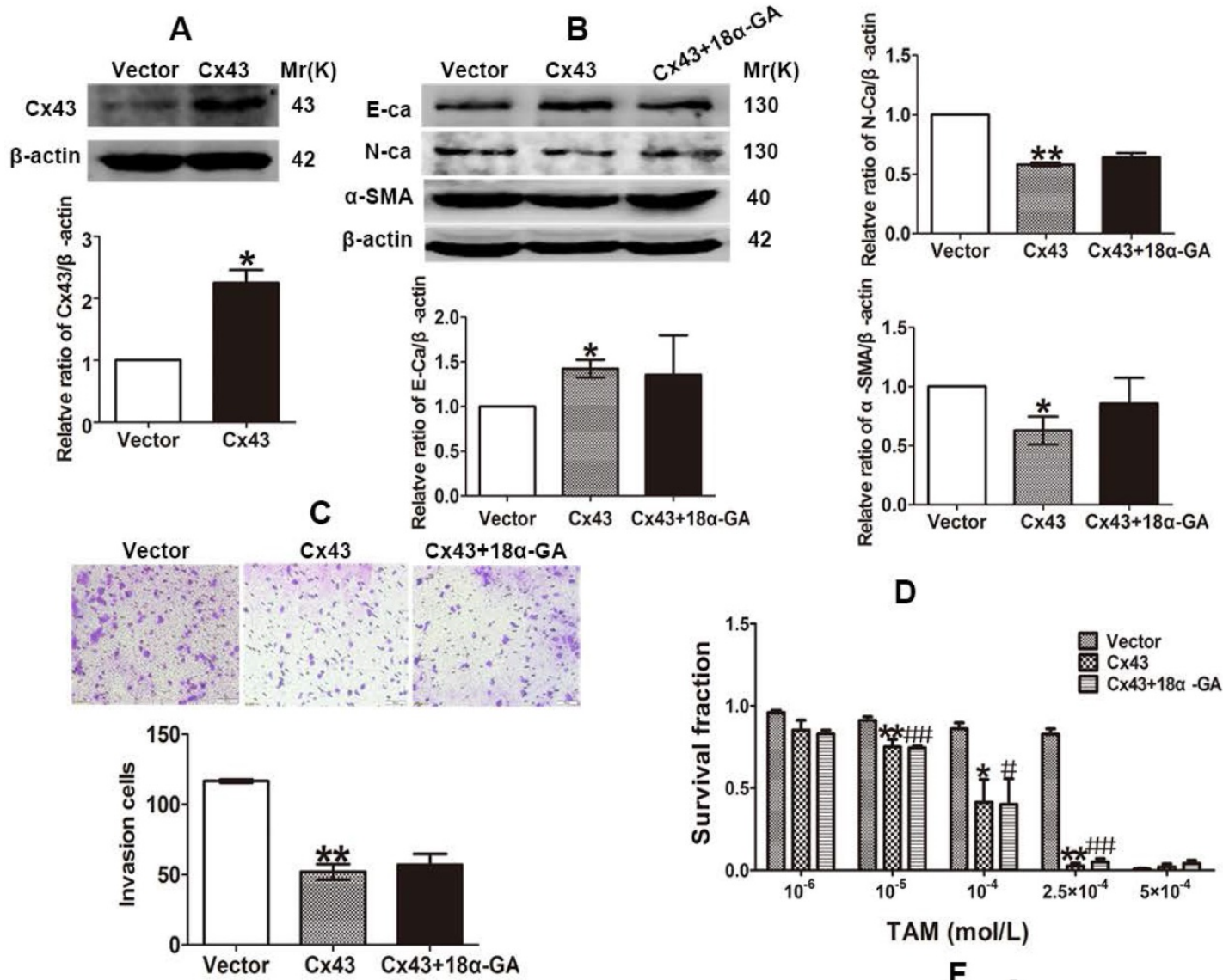

E
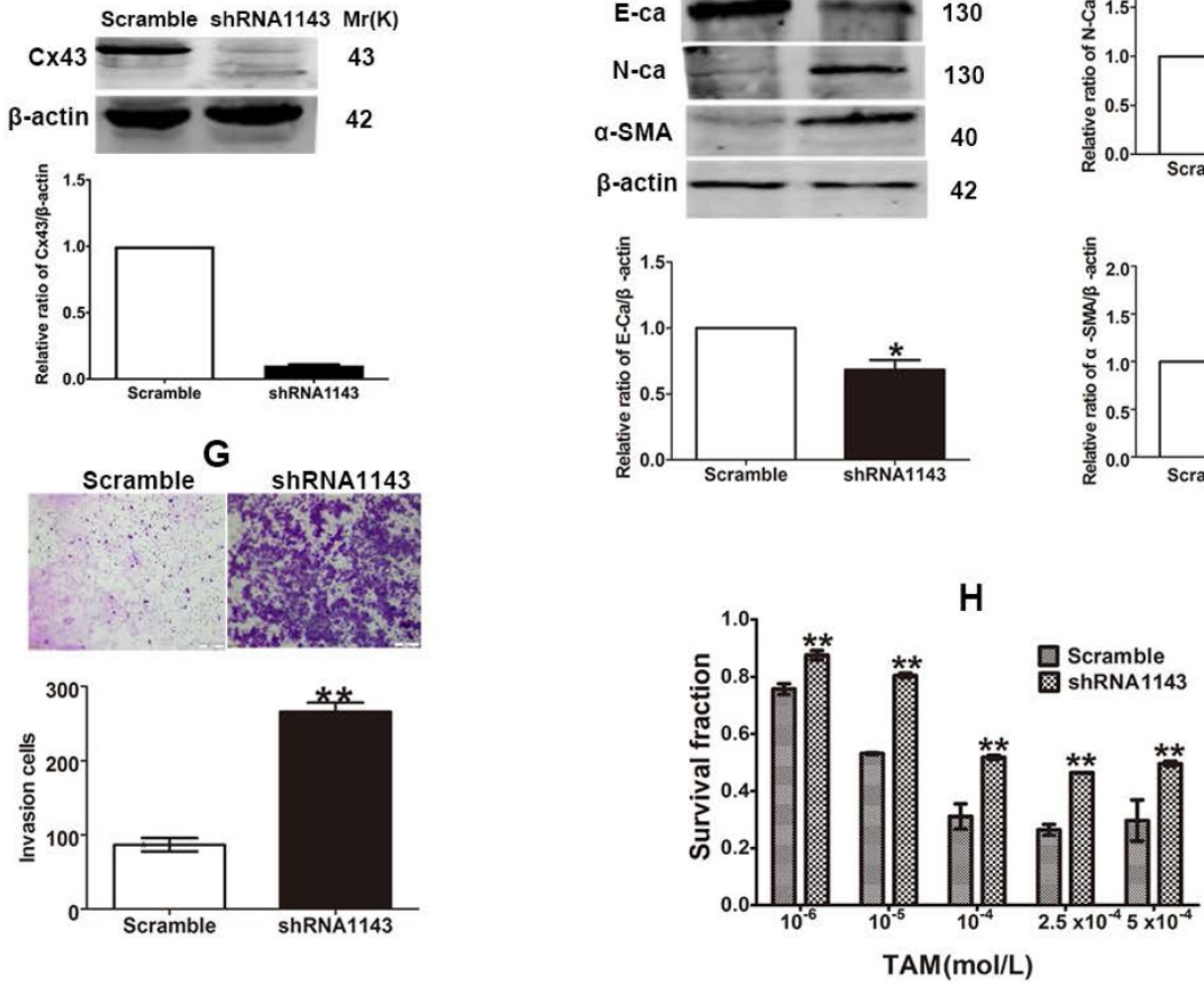

Figure 7. Cx43 reverses TAM resistance by suppressing EMT in acquired T47D/TR cells in a GJIC-independent manner. (A, B) Effect of Cx43 overexpression on E-ca, N-ca and $\alpha$-SMA expression. (C) Effect of $C \times 43$ overexpression on invasive ability. Original magnification, $\times 100$. (D) Effect of Cx43 overexpression on TAM sensitivity. Data are mean $\pm S D$ from 3 independent experiments. $* P<0.05, * * P<0.01$ versus vector group, $\# P<0.05$, \#P<0.01, versus Cx43 group. (E, F) Effect of Cx43 knockdown on $E$-ca, $N$-ca and $\alpha-S M A$ expression. $(G)$ Effect of $C \times 43$ knockdown on invasive ability. Original magnification, $\times 100 .(H)$ Effect of Cx43 knockdown on TAM sensitivity. Data are mean \pm SD from 3 independent experiments. $* P<0.05, * * P<0.01$ versus scramble group. 
A
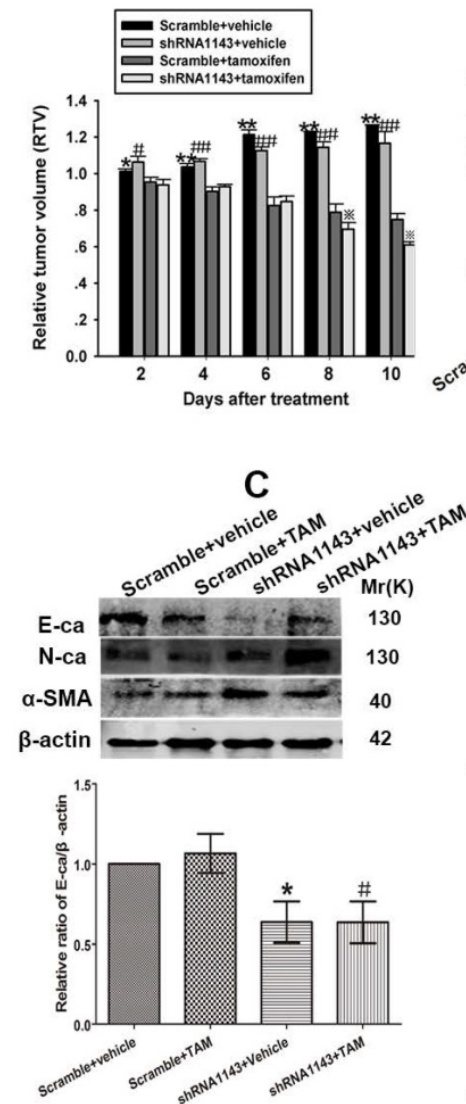

B
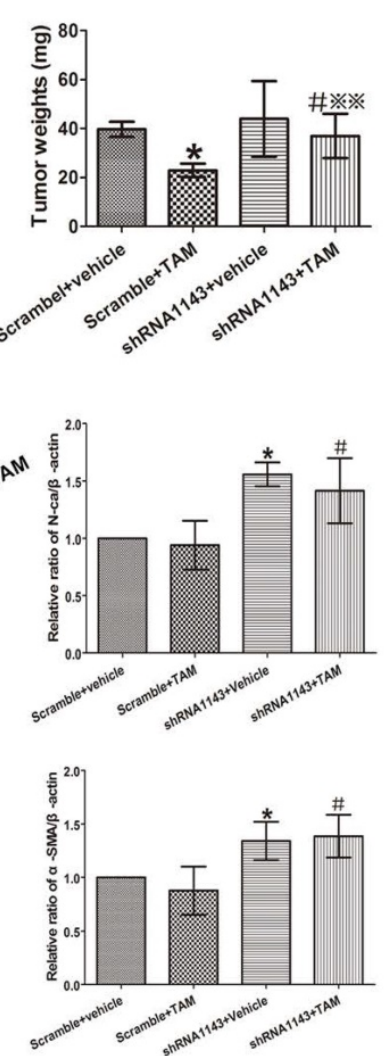

D

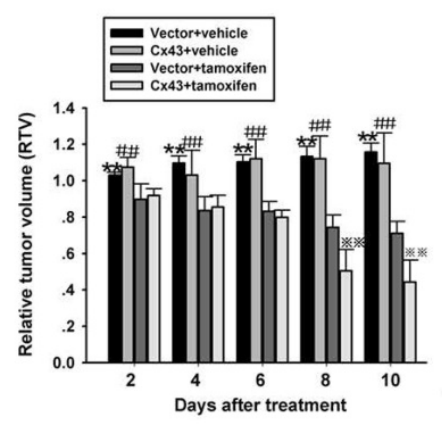

$F$
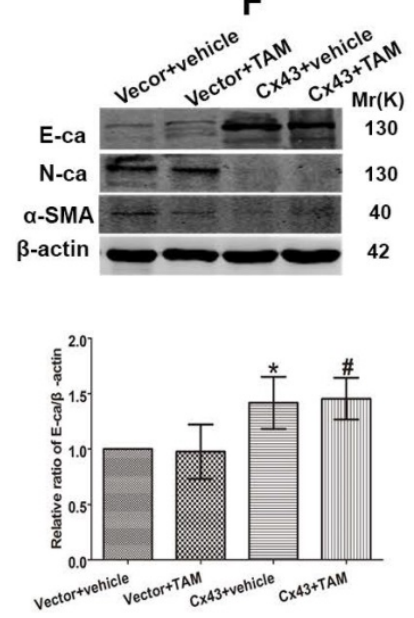

E
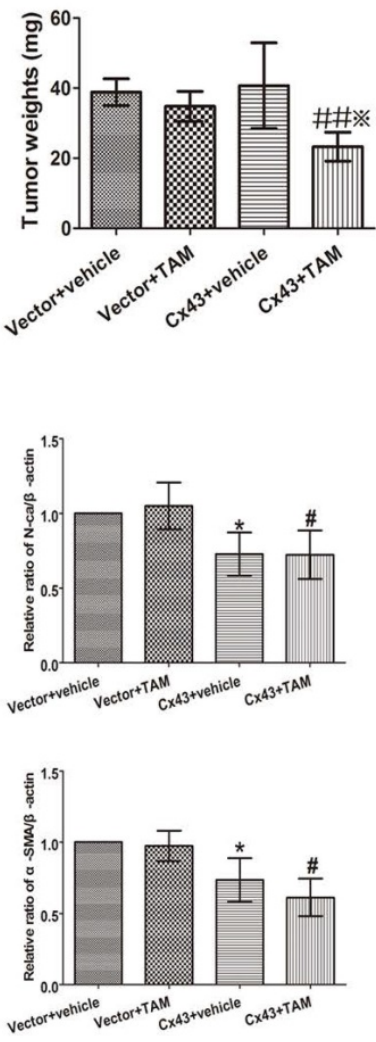

Figure 8. Effect of Cx43 expression on TAM sensitivity in xenograft tumors derived from T47D/TR and T47D/TS cells. (A) Effect of Cx43 depletion on the relative tumor volume (RTV) of xenograft transplanted by T47D/TS cells after TAM treatment. Data are mean \pm SD from $4-8$ independent experiments. $* P<0.05$, $* * P<0.01$ versus scramble+TAM group, $\# P<0.05, \# P<0.01$ versus shRNA1 143+TAM group, $* P<0.05$ versus scramble+TAM group. (B) Effect of $C \times 43$ depletion on tumor weights of $x e n o g r a f t$ transplanted by T47D/TS cells after TAM treatment. (C) Expression of E-ca, N-ca and $\alpha$-SMA of T47D/TS cells transplanted xenograft after TAM treatment. Data are mean \pm SD from 4-8 independent experiments. ${ }^{*} P<0.05$ versus scramble+vehicle group, $\# P<0.05$ versus shRNA1143+vehicle group, $* * P<0.01$ versus scramble+TAM group. (D) Effect of $C \times 43$ overexpression on the RTV of xenograft transplanted by T47D/TR cells after TAM treatment. Data are mean \pm SD from $4-8$ independent experiments. $* * P<0.01$ versus vector+TAM group, $\# P<0.01$ versus $C x 43+T A M$ group, $* * P<0.01$ versus vector+TAM group. (E) Effect of $C x 43$ overexpression on tumor weights of xenograft transplanted by T47D/TR cells after TAM treatment. \#P<0.01 versus vector+TAM group, ${ }^{*} P<0.05$ versus $C x 43+$ vehicle group. (F) Expression of E-ca, N-ca and $\alpha-S M A$ of $x$ enograft-transplanted T47D/TR cells after TAM treatment. Data are mean \pm SD from 4-8 independent experiments. $* P<0.05$ versus vector+vehicle group, $\# P<0.05$, $\# P<0.01$ versus $C \times 43+v e h i c l e ~ g r o u p$, $* P<0.05$ versus vector+TAM group.

\section{Cx43 depletion induces TAM insensitivity in T47D/TS cells transplanted xenograft tumor model}

To investigate the role of $\mathrm{Cx} 43$ in modulating TAM sensitivity in vivo, Cx43-deficient and its scramble T47D/TS cells were transplanted into $\mathrm{BALB} / \mathrm{c}$-nude mice. The xenograft-bearing mice were administrated with TAM citrate $(30 \mathrm{mg} / \mathrm{kg}$ per day, gavaged orally) for 10 days, and the mean RTV was calculated for each treatment group. As shown in Fig. $8 \mathrm{~A}$, TAM treatment suppressed tumor growth in both Cx43-deficient and scramble xenografts. Importantly, the inhibitory effects of TAM on the scramble xenografts were more dramatic due to a substantial decrease in their mean RTV and tumor weights, than Cx43-deficient xenografts (Fig. 8A and B). Meanwhile, the expression of EMT biomarkers were detected after treatment. The results showed that E-ca expression was significantly attenuated, while $\mathrm{N}$-ca and a-SMA expressions were greatly increased in Cx43-deficient xenografts compared with scramble xenografts (Fig. $8 \mathrm{C})$. The results indicate that $\mathrm{C} \times 43$ depletion facilitates TAM insensitivity via inducing EMT in vivo.

\section{Cx43 overexpression reverses TAM resistance in T47D/TR cells transplanted xenograft tumor model}

We next explored the role of Cx43 in reversing TAM resistance in vivo. Cx43-overexpressed and its control T47D/TR cells were used to establish xenograft bearing mouse model. The mice were gavaged orally with $30 \mathrm{mg} / \mathrm{kg}$ TAM citrate for 10 days. As illustrated in Fig. 8D, TAM treatment prohibited tumor growth in both $\mathrm{Cx} 43$-overexpressed and control xenografts. Notably, Cx43-overexpressed xenografts exhibited more sensitivity to TAM as compared to control xenografts due to greatly reduced mean RTV and tumor weights in Cx43overexpressed xenografts than control xenografts 
(Fig. 8D and E). Additionally, Cx43 overexpression could significantly augment E-ca expression and inhibit N-ca and a-SMA expression in xenograft tumors (Fig. 8F). These results suggest that $\mathrm{C} \times 43$ overexpression reverses TAM resistance via inhibiting EMT in vivo.

\section{Reciprocal regulation between $\mathrm{Cx} 43$ and PI3K/Akt pathway is involved in Cx43-mediated repression in EMT and TAM resistance}

According to the above results, we became interested in investigating the underlying molecular mechanism of Cx43-mediated repression of EMT and reversion of TAM resistance in breast cancer cells. It has been proven that PI3K/Akt pathway plays a vital role in activating EMT and drug resistance in malignancies [33, 34]. There are reports showing that Cx43 could activate PI3K/Akt signaling and PI3K/ Akt signaling could increase $\mathrm{C} \times 43$ phosphorylation in cardiomyocytes $[35,36]$. Thus, we hypothesized that there may be a mutual regulation between $\mathrm{C} \times 43$ and PI3K/Akt pathway in EMT and acquired TAM resistance. As illustrated in Fig. 9A, p-Akt expression was remarkably increased in acquired T47D/TR cells compared with their parental cells. Overexpressing Cx43 could significantly reduce Akt phosphorylation, while depleting $\mathrm{Cx} 43$ expression could greatly elevate Akt phosphorylation in T47D/TR cells (Fig. 9B and C). These results indicate that $\mathrm{C} \times 43$ is sufficient to retard PI3K/Akt signaling activation.

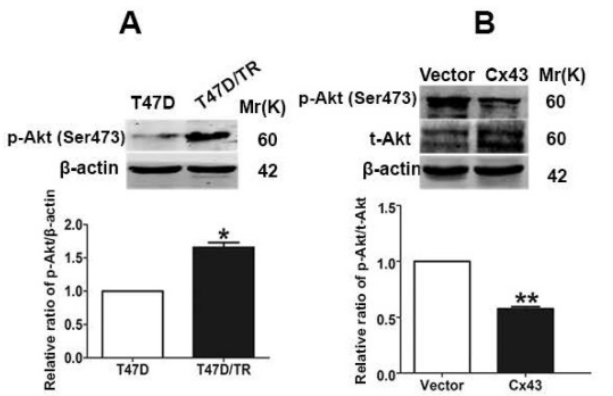

E


H

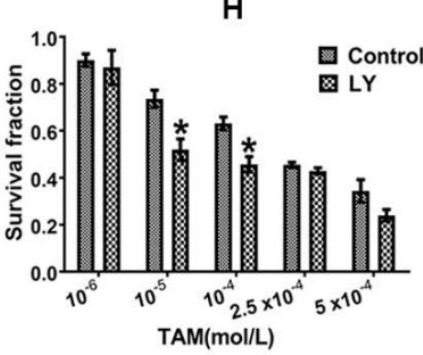

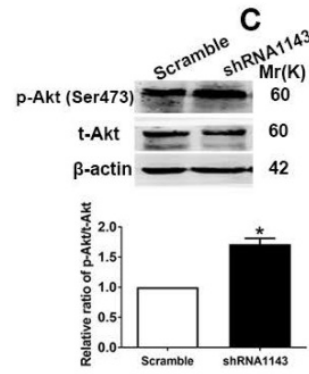

$$
\text { F }
$$
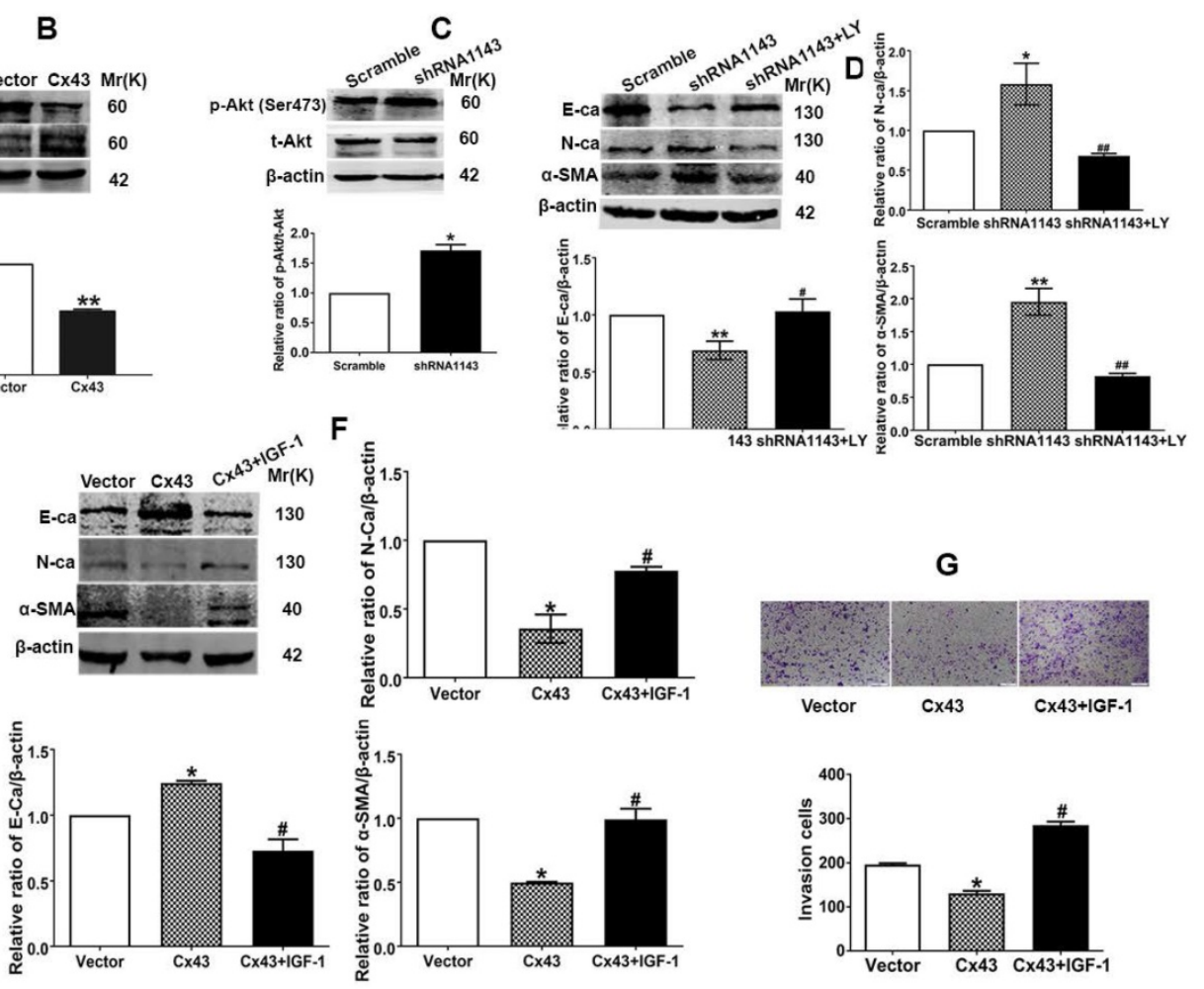

J

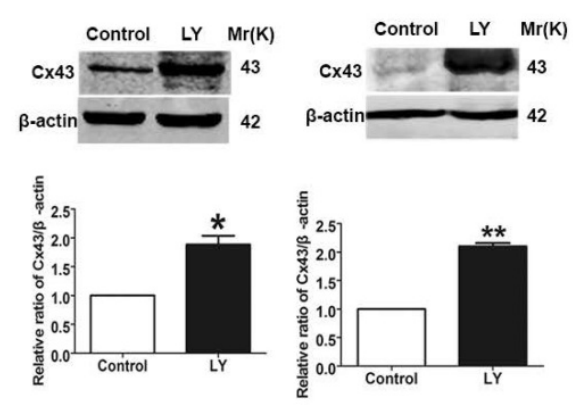

Figure 9. Reciprocal regulation between $\mathrm{Cx} 43$ and PI3K/Akt pathway is involved in Cx43-mediated repression in EMT and TAM resistance. (A) Akt activity of T47D/TR and parental cells. (B, C) Effects of Cx43 overexpression and knockdown on Akt activity in T47D/TR cells. (D) Effect of LY294002 on E-ca, N-ca and a-SMA expression in Cx43-depleted T47D/TR cells. (E) Effect of LY294002 on invasive ability in Cx43-depleted T47D/TR cells. Original magnification, $\times 100$. (F) Effect of LY294002 on TAM sensitivity in Cx43-depleted T47D/TR cells. (G) Effect of IGF-1 on E-ca, N-ca and a-SMA expression in Cx43-overexpressed T47D/TR cells. (H) Effect of IGF-1 on invasive ability in Cx43overexpressed T47D/TR cells. Original magnification, $\times 100$. (I) Effect of IGF-1 on TAM sensitivity in Cx43- overexpressed T47D/TR cells. (J, K) Effect of Akt activity on Cx43 expression in T47D/TR and T47D/TS cells, respectively. Data are mean \pm SD from 3 independent experiments. $* P<0.05$, $* * P<0.01$ versus control, vector or scramble group, $\# P<0.05, \ldots P<0.01$ versus shRNA1143 group. 
We next explored the role of PI3K/Akt signaling in Cx43-mediated EMT repression. As illustrated in Fig. 9D and E, treatment of Cx43-depleted T47D/TR cells with a specific PI3K/Akt signaling inhibitor LY294002 could attenuate the facilitating effects of Cx43 depletion on EMT, as represented by the elevated E-ca expression, decreased N-ca and a-SMA expression and attenuated invasive capability as compared to LY294002-untreated cells. Whereas, pretreatment of Cx43-overexpressed T47D/TR cells with PI3K/Akt signaling activator IGF-1 remarkably eliminated the inhibitory effects of Cx43 overexpression on EMT (Fig. 9F and G). Furthermore, LY294002-treated Cx43-depleted T47D/TR cells exhibited more sensitivity to TAM compared with LY294002-untreated cells (Fig. 9H), while IGF-1treated Cx43-overexpressed T47D/TR cells showed less TAM sensitivity compared to IGF-1-untreated cells (Fig. 9I). The results imply that PI3K/Akt signaling is required for $\mathrm{C} \times 43$-mediated reversion of EMT and TAM resistance.

We then investigated whether PI3K/Akt pathway was involved in Cx43 expression. As shown in Fig. 9J and K, treatment with LY294002 resulted in substantial increased Cx43 expression in both T47D/TR and T47D/TS cells, indicating that $\mathrm{PI} 3 \mathrm{~K} /$ Akt signaling is sufficient to attenuate Cx43 expression.

Taken together, the above findings suggest that the functional interplay between Cx43 and PI3K/Akt pathway contributes to the acquired TAM resistance in breast cancer cells via the induction of EMT.

\section{Cx43 and c-Src interaction leads to inactivation of PI3K/Akt signaling and attenuation of EMT and TAM resistance}

We then became interested in investigating the mechanism of which Cx43 retards PI3K/Akt pathway in breast cancer cells. It has been documented that the intracellular carboxy tail of Cx43 interacts with multiple signaling and scaffolding proteins, thereby modulating the intercellular signaling [37]. We next sought to predict the downstream target protein that could potentially interact with $\mathrm{C} \times 43$ and preferably associate with regulation of PI3K/Akt pathway and TAM resistance using the STRING database (http:// string-db.org/) [38] and the Human Protein Reference Database (HPRD) (http://www.hprd.org/) [39]. We found that $\mathrm{C} \times 43$ could potentially interact with c-Src, a member of the Src family of non-receptor tyrosine kinases, via its SH3-binding domain. c-Src has been reported to play vital roles in EMT, chemotherapy resistance and activate PI3K/Akt pathway [40, 41]. Therefore, we expected that the interaction between Cx43 and c-Src might play a role in the activation of
PI3K/Akt pathway, subsequently affect EMT and TAM resistance. We firstly determined the interaction of Cx43 with c-Src. The results of Co-IP assay revealed that Cx43 could interact with c-Src (Fig. 10A). Interestingly, impaired $\mathrm{C} \times 43$ and c-Src interaction was observed in T47D/TR cells than their parental T47D cells (Fig. 10A). Additionally, exogenously expressing Cx43 in T47D/TR cells suppressed the phosphorylation of c-Src (Fig. 10B), implying that Cx43 and c-Src interaction attenuates c-Src activation.

We next detected whether c-Src affects the activation of PI3K/Akt signaling in T47D/TR cells. As illustrated in Fig. 10C, treatment of Cx43-depleted T47D/TR cells with c-Src inhibitor dasatinib could attenuate the facilitating effect of $\mathrm{Cx} 43$ depletion on Akt phosphorylation, indicating that c-Src participates in Cx43-mediated inhibition of Akt activation.

We then examined whether c-Src participated in Cx43-meidated repression of EMT and TAM resistance. As shown in Fig 10D and E, treatment of Cx43-depleted T47D/TR cells with c-Src inhibitor dasatinib could attenuate the facilitating role of $\mathrm{C} \times 43$ depletion in EMT as represented by elevated E-ca expression and decreased N-ca, a-SMA expression and invasive ability. Additionally, dasatinib-treated Cx43-depleted T47D/TR cells showed more sensitivity to TAM compared with dasatinibuntreated cells (Fig. 10F). These data indicate that c-Src is required for Cx43-mediated inhibition in EMT and TAM resistance.

In summary, the above data indicate that Cx43 and c-Src interaction suppresses c-Src activation, leading to the inactivation of PI3K/Akt signaling, thereby attenuating EMT and TAM resistance.

\section{Discussion}

Endocrine therapy plays a vital role in improving the prognosis of $\mathrm{ER}^{+}$breast cancer. TAM is an important antiestrogen drug that can improve the 5 -year overall survival and disease-free survival of $\mathrm{ER}^{+}$breast cancer patients [42]. Nevertheless, de novo and acquired TAM resistance remains a major therapeutic challenge [43]. Therefore, it is critical to identify novel therapeutic targets or more specific biomarkers predicting the therapeutic response to TAM.

Here, we characterized a critical role of Cx43 in TAM resistance. We found that $\mathrm{C} \times 43$ expression was downregulated in acquired TAM-resistant breast cancer cells. Cx43 overexpression or combination treatment with dasatinib improved TAM susceptibility, while Cx43 depletion induced TAM resistance, in T47D/TS and T47D/TR cells and xenograft tumors (Figs. 4-10). These results 

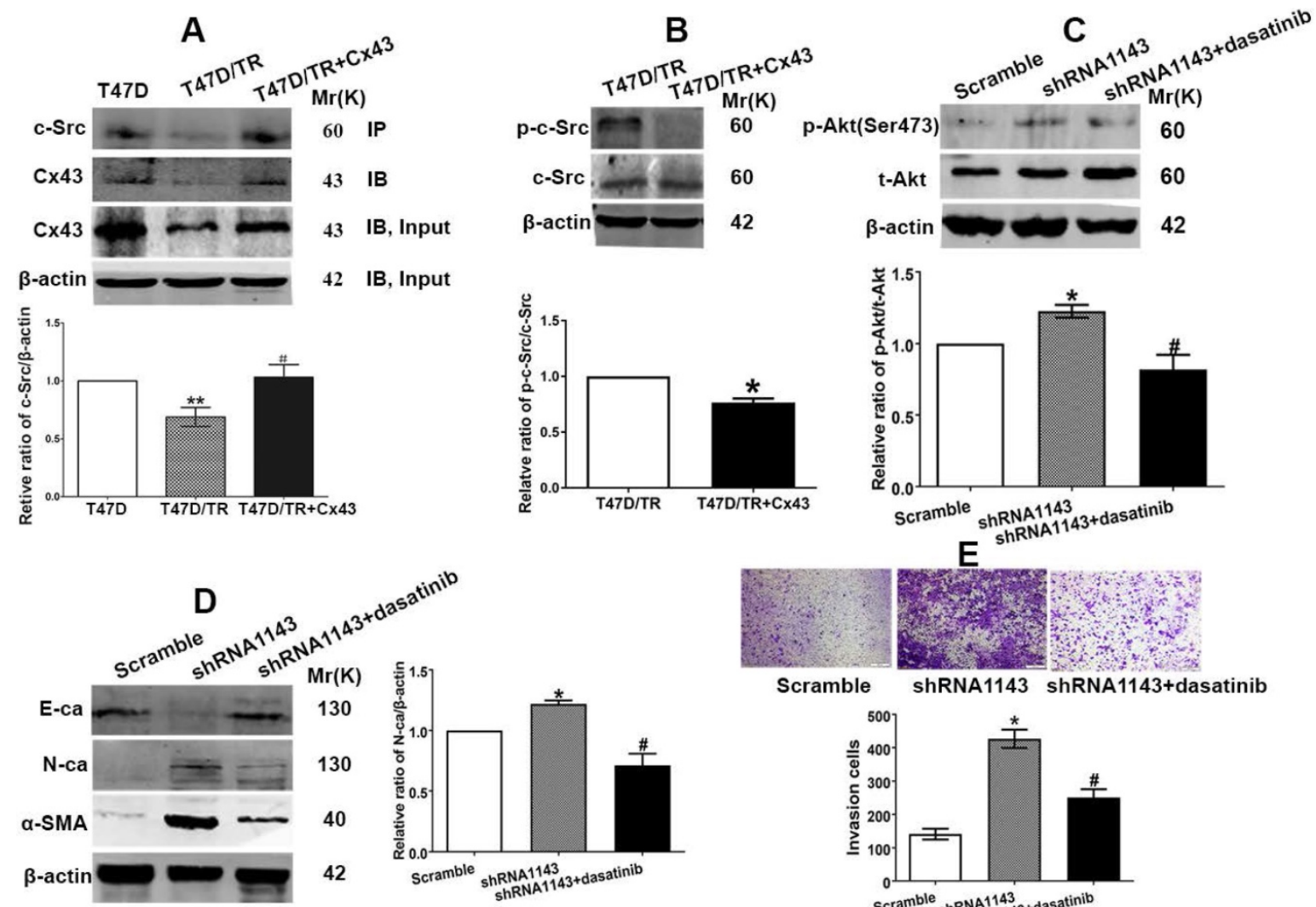

\section{E}
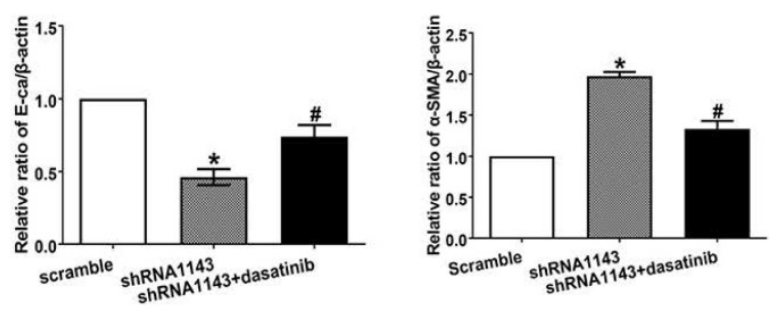

Scramble shRNA1143 shRNA1143+dasatinib
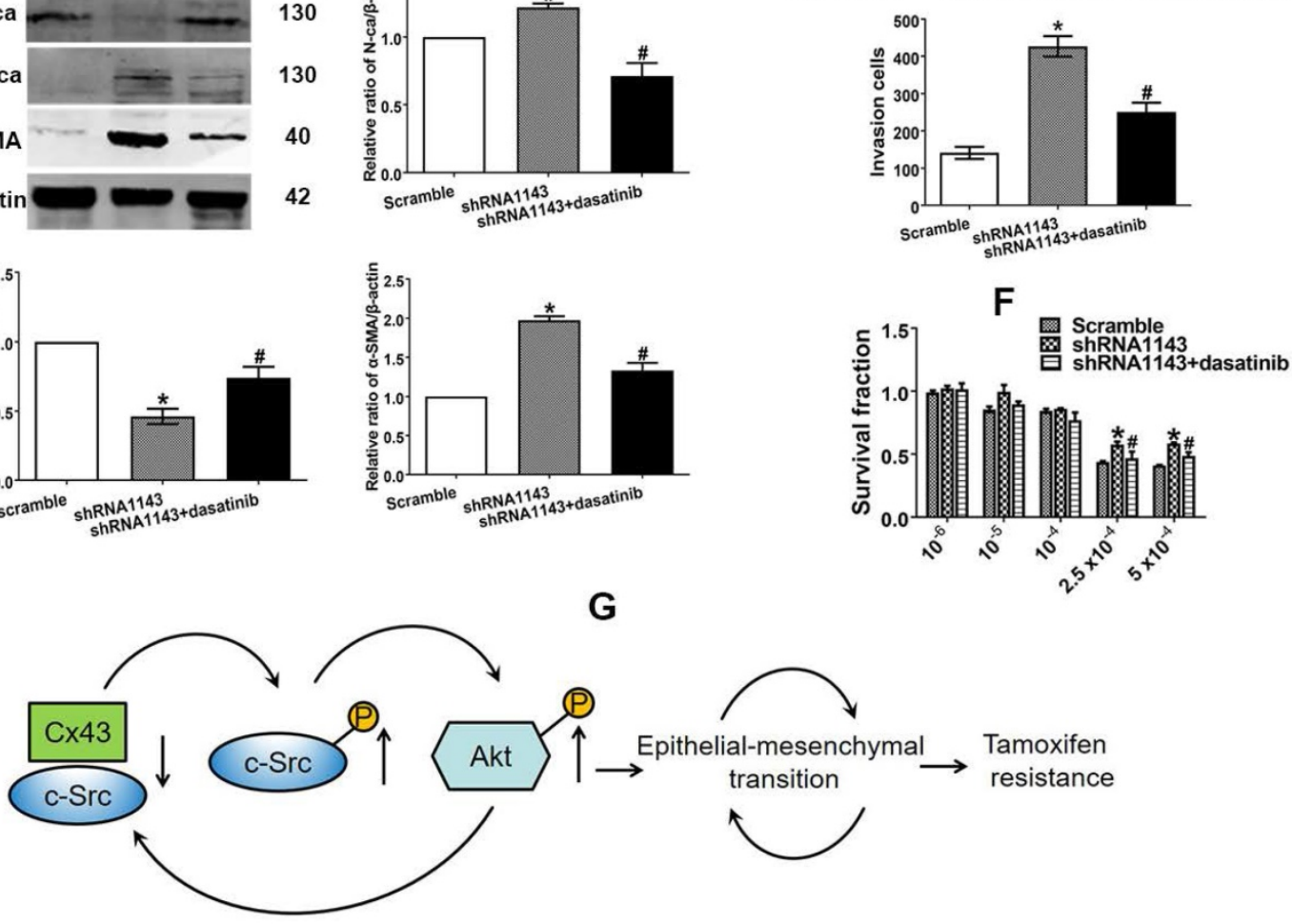

Figure 10. Cx43 and c-Src interaction leads to inactivation of PI3K/Akt signaling and attenuation of EMT and TAM resistance. (A) The interaction of Cx43 with c-Src was detected using Co-IP assay. (B) c-Src activity of T47D/TR cells. (C) Akt activity of Cx43-depleted T47D/TR cells after cells were treated with dasatinib. (D) Effect of dasatinib on $\mathrm{E}-\mathrm{ca}, \mathrm{N}$-ca and $\alpha$-SMA expression in Cx43-depeleted T47D/TR cells. (E) Effect of dasatinib on invasive ability in Cx43-depeleted T47D/TR cells. Original magnification, $\times 100$. (F) Effect of dasatinib on TAM sensitivity in Cx43-depeleted T47D/TR cells. Data are mean \pm SD from 3 independent experiments. $* P<0.05$, **P<0.01 versus T47D, T47D/TR or scramble group, $\# P<0.05, \ldots P<0.01$ versus T47D/TR or shRNA1 143 group. (G) Model of feedback loop contributing to TAM resistance. The regulatory circuit impedes Cx43 expression, leading to an impairment of the interaction of $\mathrm{Cx} 43$ with c-Src and subsequently activation of PI3K/Akt pathway, which further retards Cx43 expression, thereby augmenting EMT and TAM resistance in breast cancer.

demonstrate that $\mathrm{C} \times 43$ could serve as an attractive target for overcoming TAM resistance and suggest that therapeutic strategies designed to maintain or enhance Cx43 expression may augment the efficacy of TAM treatment.

In the present study, we also found that high Cx43 expression was correlated with low TNM stage, low lymph node metastasis and high 10-year overall survival and relapse-frees survival in the entire patient cohort and the subtypes of $\mathrm{ER}^{+}$, basal and luminal A (Fig. 1B-K), indicating that Cx43 serves as an independent prognostic marker for breast cancer patients. These findings are consistent with a recent study that elevating Cx43 protein levels were linked 
with significantly improved metastasis-free survival [44]. Notably, data mining analysis of TCGA and several GEO datasets in the cohort of breast cancer patients who received TAM monotherapy revealed that high Cx43 expression was highly associated with improved outcome in relapse free survival (Fig. 1J and $\mathrm{K}$, Fig. 2), suggesting that $\mathrm{C} \times 43$ may serve as a potential prognostic marker for the therapeutic response to TAM treatment.

The present study makes clear that there is a significant Cx43-dependent component of TAM susceptibility, indicating that $\mathrm{C} \times 43$ expression in breast cancer is an important determiner of the clinical response to TAM treatment. Agents affecting Cx43 expression may influence the clinical response to TAM-based endocrinotherapy. It has been proven that several agents, including all-trans retinoic acid (ATRA) and resveratrol, increase the expression of Cxs including Cx43, causing an enhancement of antineoplastic efficacy $[45,46]$. Thus, the efficacy of TAM therapy could be increased in the event of TAM treatment used concurrently with these agents in clinical settings. It should be noted, however, that several platinum-based chemotherapeutics, such as cisplatin and oxaliplatin, have been reported to inhibit Cx43 expression [47]. Therefore, if TAM therapy is used concurrently with platinum-based chemotherapy, the antitumor efficiency of TAM might be declined, probably leading to TAM resistance, by the suppression of Cxs expression caused by platinum-based reagents.

EMT is integral in wound healing, cell behavior and pathologically contributes to cancer progression and chemotherapeutic resistance [48]. Reports have indicated that TAM-resistant MCF-7 breast cancer cells undergo EMT phenotype [8, 49]. In the present study, we observed that T47D/TR cells exerted EMT phenotype (Fig. 3B-E and G). Induction EMT of T47D/TS cells by TGF- $\beta 1$ could attenuate TAM sensitivity (Fig. 4A and B), whereas repression EMT of T47D/TR cells by MS-275 could reverse TAM resistance (Fig. 4D and G). These results are consistent with previous studies which indicate that EMT is a key event to confer TAM resistance $[50,51]$. We next investigated whether $\mathrm{C} \times 43$ reversed TAM resistance via retarding EMT. We found that high Cx43 expression was significantly correlated with high E-ca expression and low a-SMA expression in clinical specimens (Fig. 5A and B) and Cx43 was sufficient to alleviate TAM resistance by impeding EMT in TAM-sensitive and -resistant breast cancer cells and in xenografts (Fig. 5E-G, Fig 8). These results suggest the possibility that attenuating EMT by up-regulation or maintenance of Cx43 expression may be a novel therapeutic strategy to overcome TAM resistance.
GJIC-dependent effects of Cxs, including Cx32, Cx43 and Cx26, on the sensitivity of antineoplastics have been reported in many studies [22, 52, 53]. In the present study, we observed that 18a-GA, a well-defined GJIC inhibitor, restored the inhibitory role of Cx43 in TGF- $\beta 1$-induced EMT and TAM insensitivity in TAM-sensitive cells (Fig. 5D-G), indicating a GJIC-dependent role of Cx43 in reversing TGF- $\beta 1$-induced EMT and TAM insensitivity in TAM-sensitive cells. It has been well established that GJIC-mediated effects on the sensitivity of antineoplastic agents depend on the transfer of toxic substances from one cell to an adjacent other via GJ channels [11]. It would be interesting to investigate the substances penetrating via GJ channels responsible for TGF- $\beta 1$-induced TAM sensitivity in further study. In spite of GJIC-dependent role of Cxs in chemosensitivity, GJIC-independent effects of Cxs on the resistance of antineoplastic agents have also been reported recently. For example, Cx26 protein itself, which could not form GJIC, could promote gefitinib resistance in NSCLC cells [54]. Additionally, Cx43 prohibited cisplatin resistance in a GJICindependent manner [55]. In the present study, we found that GJIC inhibitor 18a-GA could not suppress the inhibitory role of $\mathrm{Cx} 43$ in EMT and TAM sensitivity in TAM-resistant cells (Fig. 7B-D), suggesting that $\mathrm{C} \times 43$-mediated reversion of EMT and TAM resistance is independent of GJIC.

PI3K/Akt signaling-dependent EMT has been reported to facilitate resistance to chemotherapy in human multiple myeloma [56], cisplatin resistance in hepatocellular carcinoma cells [57] and TAM resistance in breast cancer cells [58]. Reports have demonstrated that $\mathrm{C} \times 43$ could activate PI3K/Akt signaling in cardiomyocytes [36] and PI3K/Akt signaling activation facilitates $\mathrm{C} \times 43$ expression in myocardial ischemia-reperfusion injury [59]. Thus, we hypothesized that there might exist a mutual regulation of $\mathrm{PI} 3 \mathrm{~K} / \mathrm{Akt}$ signaling and $\mathrm{Cx} 43$ expression in acquired endocrine treatment resistance. In the study, we found that PI3K/Akt pathway was activated in acquired T47D/TR cells (Fig. 9A). Exogenous Cx43 expression could prevent PI3K/Akt signaling activation, while $\mathrm{C} x 43$ depletion could activate Akt in T47D/TR cells (Fig. 9B and C). Moreover, suppression of PI3K/Akt by specific inhibitor LY294002 could attenuate the facilitating effects of Cx43 depletion on EMT and TAM resistance, whereas pretreatment of Cx43-overexpressed T47D/ TR cells with PI3K/Akt signaling activator IGF-1 remarkably eliminated the inhibitory effects of Cx43 overexpression on EMT and TAM resistance in acquired T47D/TR cells (Fig. 9D-I). These data suggest that PI3K/Akt pathway is required for Cx43- 
mediated inhibition of EMT and TAM resistance. Interestingly, we also found that inhibition of PI3K/Akt pathway led to decreased Cx43 expression in T47D/TR and their parental cells (Fig. 9J and K). However, the underlying mechanism was not investigated in the study. There are reported showing that activation of PI3K/Akt signaling by shear stress resulted in elevated accumulation of $\beta$-catenin in nucleus, which could elevate $C \times 43$ expression by binding to the Cx43 promoter [60]. Further study should be designed to investigate whether $\beta$-catenin was involved in the elevation of $\mathrm{C} \times 43$ expression by PI3K/Akt pathway. Collectively, these data indicate that there exists a feedback modulation between Cx43 expression and PI3K/Akt pathway, retarding Cx43 expression and activating PI3K/Akt signaling, subsequently contributing to EMT and TAM resistance in breast cancer.

The carboxy tail of $\mathrm{C} \times 43$ has been reported to contain multiple domains interacting with scaffolding proteins [61]. We then predicted the proteins that could potentially interact with Cx43 and preferably regulate PI3K/Akt pathway and TAM resistance using STRING and HPRD databases. c-Src was selected as a downstream protein of $\mathrm{C} \times 43$ since c-Src has been reported to activate PI3K/Akt pathway by directly phosphorylating its p85 regulatory subunit $[40,62]$ and participate in EMT and the resistance of chemotherapy and endocrinotherapy [63, 64]. We hypothesized that $\mathrm{C} \times 43$ and c-Src interaction might modulate c-Src activity, thereby regulating the activation of PI3K/Akt pathway. Co-IP assays directly support the interaction of $\mathrm{Cx} 43$ with c-Src (Fig. 10A). Western blotting assay revealed that overexpression of Cx43 sufficiently inhibited c-Src phosphorylation (Fig. 10B), indicating that the interaction of Cx43 with c-Src reduces c-Src activity, which is consistent with the result that $\mathrm{Cx} 43$ and c-Src interaction suppressed c-Src activity in glioma stem cells [65]. In addition, Co-IP assay revealed that Cx43 and c-Src interaction was significantly impaired in T47D/TR cells than their parental T47D cells. Moreover, inhibition of c-Src activity by dasatinib in Cx43-depelted cells attenuated the facilitating role of Cx43 depletion in EMT and TAM resistance (Fig $10 \mathrm{D} \sim \mathrm{F})$, indicating that $\mathrm{C} \times 43$ and $\mathrm{c}-\mathrm{Src}$ interaction deficit contributes to EMT and TAM resistance. We then investigated whether c-Src was involved in Cx43-mediated modulation of PI3K/Akt signaling. The results showed that suppressing c-Src activity by dasatinib attenuated the enhanced role of Cx43 depletion in Akt phosphorylation (Fig. 10C), implying the participation of c-Src in Cx43-mediated inhibition of Akt activation. Collectively, the above data indicate that $\mathrm{C} \times 43$ and c-Src interaction suppresses c-Src activation, leading to the inactivation of PI3K/Akt signaling, thereby attenuating EMT and TAM resistance.

It is worth noting that a novel feedback regulation between the interaction of $\mathrm{C} \times 43$ with c-Src and PI3K/Akt pathway conferring TAM resistance was found in the study. The regulatory circuit impedes Cx43 expression, leading to an impairment of the interaction of $\mathrm{Cx} 43$ with c-Src and subsequently activation of PI3K/Akt pathway, which further retards Cx43 expression, thereby inducing EMT and TAM resistance in breast cancer (Fig. 10G). Therefore, therapeutic strategies designed to disturb the regulatory loop may be beneficial to interrupt EMT and TAM resistance. There are reports showing that cell-penetrating peptides synthesized according to the sequences of $\mathrm{Cx} 43$ containing c-Src binding sites could reduce c-Src activity and reverse EMT in glioma stem cells [65]. Accordingly, one would expect cell-penetrating peptide to reverse EMT and TAM resistance by disturbing the feedback loop. It is necessary to investigate the role of cell-penetrating peptide in EMT and TAM sensitivity in our further studies.

In summary, the work reported here demonstrates that Cx43 deficiency confers TAM resistance by inducing EMT via activating c-Src/ PI3K/Akt pathway. This brings about several clinical considerations. Firstly, pharmacologic strategies designed to reinforce $\mathrm{C} \times 43$ function can sensitize breast cancer cells to endocrine therapy. Oppositely, factors that suppress the functionality of Cx43 may lead to endocrine treatment resistance in breast cancer, causing substantial declined therapeutic efficacy. Secondly, the assessment of $\mathrm{C} \times 43$ expression may be used to categorize patients in accordance with their benefits from TAM treatment, which may offer patients a better choice for TAM and a more personalized treatment approach.

\section{Acknowledgements}

This project was supported by Postdoctoral Science Foundation of China (No. 2016M591927), Natural Science Foundation of China (No. 81402946) and Xuzhou science and technology project under grant KC19018.

\section{Data Availability Statement}

The processed data required to reproduce these findings cannot be shared at this time as the data also forms part of an ongoing study.

\section{Competing Interests}

The authors have declared that no competing interest exists. 


\section{References}

1. Rozeboom B, Dey N, De P. ER+ metastatic breast cancer: past, present, and a prescription for an apoptosis-targeted future. Am J Cancer Res. 2019; 9: 2821-31.

2. Caparica R, Brandao M, Piccart M. Systemic treatment of patients with early breast cancer: recent updates and state of the art. Breast. 2019; 48 Suppl 1: S7-S20.

3. Thorat MA, Balasubramanian R. Breast cancer prevention in high-risk women. Best Pract Res Clin Obstet Gynaecol. 2020; 65: 18-31.

4. Hua W, Ten Dijke P, Kostidis S, Giera M, Hornsveld M. TGFbeta-induced metabolic reprogramming during epithelial-to-mesenchymal transition in cancer. Cell Mol Life Sci. 2020; 77: 2103-23.

5. Du B, Shim JS. Targeting Epithelial-Mesenchymal Transition (EMT) to Overcome Drug Resistance in Cancer. Molecules. 2016; 21

6. Tong M, Gao M, Xu Y, Fu L, Li Y, Bao X, et al. SHR-A1403, a novel c-mesenchymal-epithelial transition factor (c-Met) antibody-drug conjugate, overcomes AZD9291 resistance in non-small cell lung cancer cells overexpressing c-Met. Cancer Sci. 2019; 110: 3584-94.

7. Han Q, Cheng P, Yang H, Liang H, Lin F. miR-146b Reverses epithelialmesenchymal transition via targeting PTP1B in cisplatin-resistance human lung adenocarcinoma cells. J Cell Biochem. 2019.

8. Xiao Y, Xie Q, Qin Q, Liang Y, Lin H, Zeng. Upregulation of SOX11 enhances tamoxifen resistance and promotes epithelial-to-mesenchymal transition via slug in MCF-7 breast cancer cells. J Cell Physiol. 2020; 235: 7295-308.

9. Liang YK, Zeng, Xiao YS, Wu Y, Ouyang YX, Chen M, et al. MCAM/CD146 promotes tamoxifen resistance in breast cancer cells through induction of epithelial-mesenchymal transition, decreased ERalpha expression and AKT activation. Cancer Lett. 2017; 386: 65-76.

10. Tian M, Schiemann WP. TGF-beta Stimulation of EMT Programs Elicits Non-genomic ER-alpha Activity and Anti-estrogen Resistance in Breast Cancer Cells. J Cancer Metastasis Treat. 2017; 3: 150-60.

11. Herve JC, Derangeon M. Gap-junction-mediated cell-to-cell communication. Cell and tissue research. 2013; 352: 21-31.

12. Zefferino R, Piccoli C, Gioia SD, Capitanio N, Conese M. Gap Junction Intercellular Communication in the Carcinogenesis Hallmarks: Is This a Phenomenon or Epiphenomenon? Cells. 2019; 8.

13. Sadr-Eshkevari P, Ashnagar S, Rashad A, Dietz M, Jackowski J, Abdulazim A, et al. Bisphosphonates and connexin 43: a critical review of evidence. Cell communication \& adhesion. 2014; 21: 241-7.

14. Grek CL, Sheng Z, Naus CC, Sin WC, Gourdie RG, Ghatnekar GG. Novel approach to temozolomide resistance in malignant glioma: connexin43directed therapeutics. Curr Opin Pharmacol. 2018; 41: 79-88.

15. Su YJ, Zhang JX, Li SM, Tan XH, Huang JA. Relationship of vasculogenic mimicry, SphK1 expression, and Cx43 expression to metastasis and prognosis in colorectal cancer. Int J Clin Exp Pathol. 2018; 11: 5290-9.

16. Hwang KD, Bak MS, Kim SJ, Rhee S, Lee YS. Restoring synaptic plasticity and memory in mouse models of Alzheimer's disease by PKR inhibition. Mol Brain. 2017; 10: 57

17. Grek CL, Rhett JM, Bruce JS, Abt MA, Ghatnekar GS, Yeh ES. Targeting connexin 43 with alpha-connexin carboxyl-terminal (ACT1) peptide enhances the activity of the targeted inhibitors, tamoxifen and lapatinib, in breast cancer: clinical implication for ACT1. BMC Cancer. 2015; 15: 296.

18. Zhuang W, Ge X, Yang S, Huang M, Zhuang W, Chen P, et al. Upregulation of IncRNA MEG3 Promotes Osteogenic Differentiation of Mesenchymal Stem Cells From Multiple Myeloma Patients By Targeting BMP4 Transcription. Stem cells. 2015; 33: 1985-97.

19. Mao LJ, Yang CH, Fan L, Gao P, Yang DR, Xue BX, et al. SATB1 promotes prostate cancer metastasis by the regulation of epithelial-mesenchymal transition. Biomed Pharmacother. 2016; 79: 1-8.

20. Jiang Q, Pan D, Yang Y, Hu Y, Fang L, Shang P, et al. Luteolin Regulates Macrophage Polarization via the PI3K/Akt Pathway to Inhibit the Apoptosis Stimulated by Angiotensin II. Curr Pharm Biotechnol. 2018; 19: 428-37.

21. Mei PJ, Bai J, Miao FA, Li ZL, Chen C, Zheng JN, et al. Relationship between expression of XRCC1 and tumor proliferation, migration, invasion, and angiogenesis in glioma. Invest New Drugs. 2018

22. Wu DP, Lin TY, Bai LR, Huang JL, Zhou Y, Zhou N, et al. Enhanced phototoxicity of photodynamic treatment by Cx26-composed GIIC via ROScalcium- and lipid peroxide-mediated pathways. Journal of biophotonics. 2017; 10: 1586-96.

23. Hou PF, Jiang T, Chen F, Shi PC, Li HQ, Bai J, et al. KIF4A facilitates cell proliferation via induction of p21-mediated cell cycle progression and promotes metastasis in colorectal cancer. Cell death \& disease. 2018; 9: 477.

24. Hou P, Li L, Chen F, Chen Y, Liu H, Li J, et al. PTBP3-Mediated Regulation of ZEB1 mRNA Stability Promotes Epithelial-Mesenchymal Transition in Breast Cancer. Cancer research. 2018; 78: 387-98.

25. Shan HX, Hou PF, Zhang M, Li LT, Pan Y, Chen F, et al. PTBP1 knockdown in renal cell carcinoma inhibits cell migration, invasion and angiogenesis in vitro and metastasis in vivo via the hypoxia inducible factor-1 pathway. Int J Oncol. 2018; 52: 1613-22.

26. Zhang D, Li X, Hu Y, Jiang H, Wu Y, Ding $Y$, et al. Tabersonine attenuates lipopolysaccharide-induced acute lung injury via suppressing TRAF6 ubiquitination. Biochem Pharmacol. 2018; 154: 183-92.

27. Goswami $\mathrm{CP}$, Nakshatri H. PROGgeneV2: enhancements on the existing database. Bmc Cancer. 2014; 14.
28. Gakhar G, Hua DH, Nguyen TA. Combinational treatment of gap junctional activator and tamoxifen in breast cancer cells. Anticancer Drugs. 2010; 21: 77-88.

29. Carystinos GD, Kandouz M, Alaoui-Jamali MA, Batist G. Unexpected induction of the human connexin 43 promoter by the ras signaling pathway is mediated by a novel putative promoter sequence. Mol Pharmacol. 2003; 63: 821-31.

30. Shah P, Gau Y, Sabnis G. Histone deacetylase inhibitor entinostat reverses epithelial to mesenchymal transition of breast cancer cells by reversing the repression of E-cadherin. Breast cancer research and treatment. 2014; 143: 99-111.

31. Schech A, Kazi A, Yu S, Shah P, Sabnis G. Histone Deacetylase Inhibitor Entinostat Inhibits Tumor-Initiating Cells in Triple-Negative Breast Cancer Cells. Molecular cancer therapeutics. 2015; 14: 1848-57.

32. Aasen T, Mesnil M, Naus CC, Lampe PD, Laird DW. Gap junctions and cancer: communicating for 50 years. Nature reviews Cancer. 2017; 17: 74.

33. Ma Z, Lou S, Jiang Z. PHLDA2 regulates EMT and autophagy in colorectal cancer via the PI3K/AKT signaling pathway. Aging (Albany NY). 2020; 12: 7985-8000.

34. Wang J, Zhu H, Huang L, Zhu X, Sha J, Li G, et al. Nrf2 signaling attenuates epithelial-to-mesenchymal transition and renal interstitial fibrosis via PI3K/Akt signaling pathways. Exp Mol Pathol. 2019; 111: 104296.

35. Batra N, Riquelme MA, Burra S, Kar R, Gu SM, Jiang JX. Direct Regulation of Osteocytic Connexin 43 Hemichannels through AKT Kinase Activated by Mechanical Stimulation. J Biol Chem. 2014; 289: 10582-91.

36. Ishikawa S, Kuno A, Tanno M, Miki T, Kouzu H, Itoh $\mathrm{T}$, et al. Role of connexin-43 in protective PI3K-Akt-GSK-3beta signaling in cardiomyocytes. American journal of physiology Heart and circulatory physiology. 2012; 302: H2536-44

37. Leithe E, Mesnil M, Aasen T. The connexin 43 C-terminus: A tail of many tales. Biochim Biophys Acta Biomembr. 2018; 1860: 48-64.

38. Szklarczyk D, Gable AL, Lyon D, Junge A, Wyder S, Huerta-Cepas J, et al. STRING v11: protein-protein association networks with increased coverage, supporting functional discovery in genome-wide experimental datasets. Nucleic Acids Res. 2019; 47: D607-D13.

39. Keshava Prasad TS, Goel R, Kandasamy K, Keerthikumar S, Kumar S, Mathivanan S, et al. Human Protein Reference Database--2009 update. Nucleic Acids Res. 2009; 37: D767-72.

40. Ke LR, Xiang YQ, Guo X, Lu JP, Xia WX, Yu YH, et al. c-Src activation promotes nasopharyngeal carcinoma metastasis by inducing the epithelial-mesenchymal transition via PI3K/Akt signaling pathway: a new and promising target for NPC. Oncotarget. 2016; 7: 28340-55.

41. Parseghian CM, Parikh NU, Wu JY, Jiang ZQ, Henderson L, Tian F, et al. Dual Inhibition of EGFR and c-Src by Cetuximab and Dasatinib Combined with FOLFOX Chemotherapy in Patients with Metastatic Colorectal Cancer. Clin Cancer Res. 2017; 23: 4146-54.

42. Rani A, Stebbing J, Giamas G, Murphy J. Endocrine Resistance in Hormone Receptor Positive Breast Cancer-From Mechanism to Therapy. Front Endocrinol. 2019; 10.

43. Mills JN, Rutkovsky AC, Giordano A. Mechanisms of resistance in estrogen receptor positive breast cancer: overcoming resistance to tamoxifen/ aromatase inhibitors. Curr Opin Pharmacol. 2018; 41: 59-65.

44. Chasampalioti M, Green AR, Ellis IO, Rakha EA, Jackson AM, Spendlove I, et al. Connexin 43 is an independent predictor of patient outcome in breast cancer patients. Breast cancer research and treatment. 2019; 174: 93-102.

45. Kong H, Liu X, Yang L, Qi K, Zhang H, Zhang J, et al. All-trans retinoic acid enhances bystander effect of suicide gene therapy in the treatment of breast cancer. Oncology reports. 2016; 35: 1868-74.

46. Cheng YJ, Chang MY, Chang WW, Wang WK, Liu CF, Lin ST, et al. Resveratrol Enhances Chemosensitivity in Mouse Melanoma Model Through Connexin 43 Upregulation. Environ Toxicol. 2015; 30: 877-86.

47. Wang Q, You T, Yuan D, Han X, Hong X, He B, et al. Cisplatin and oxaliplatin inhibit gap junctional communication by direct action and by reduction of connexin expression, thereby counteracting cytotoxic efficacy. J Pharmacol Exp Ther. 2010; 333: 903-11.

48. Navas T, Kinders RJ, Lawrence SM, Ferry-Galow KV, Borgel S, Hollingshead MG, et al. Clinical Evolution of Epithelial-Mesenchymal Transition in Human Carcinomas. Cancer Res. 2020; 80: 304-18.

49. Gao Y, Zhang W, Liu C, Li G. miR-200 affects tamoxifen resistance in breast cancer cells through regulation of MYB. Sci Rep. 2019; 9: 18844.

50. Wang Q, Gun M, Hong XY. Induced Tamoxifen Resistance is Mediated by Increased Methylation of E-Cadherin in Estrogen Receptor-Expressing Breast Cancer Cells. Sci Rep. 2019; 9: 14140.

51. Luqmani YA, Alam-Eldin N. Overcoming Resistance to Endocrine Therapy in Breast Cancer: New Approaches to a Nagging Problem. Med Princ Pract. 2016; 25 Suppl 2: 28-40.

52. Yu BB, Dong SY, Yu ML, Jiang GJ, Ji J, Tong XH. Total flavonoids of litsea coreana enhance the cytotoxicity of oxaliplatin by increasing gap junction intercellular communication. Biological \& pharmaceutical bulletin. 2014; 37: 1315-22.

53. Huang F, Li S, Gan X, Wang R, Chen Z. Propofol inhibits gap junctions by attenuating sevoflurane-induced cytotoxicity against rat liver cells in vitro. European journal of anaesthesiology. 2014; 31: 219-24. 
54. Wang R, Wei RB, Yang Y, Wang N, Huang MJ, Cao CM, et al. Effect of probucol on insulin resistance in patients with non-diabetic chronic kidney disease. J Geriatr Cardiol. 2015; 12: 521-7.

55. Kim J, Yoon H, Chung DE, Brown JL, Belmonte KC, Kim J. miR-186 is decreased in aged brain and suppresses BACE1 expression. J Neurochem. 2016; 137(3): 436-45.

56. Wang L, Lin N, Li Y. The PI3K/AKT signaling pathway regulates ABCG2 expression and confers resistance to chemotherapy in human multiple myeloma. Oncol Rep. 2019; 41: 1678-90.

57. Wang N, Hou MS, Zhan Y, Shen XB, Xue HY. MALAT1 promotes cisplatin resistance in cervical cancer by activating the PI3K/AKT pathway. Eur Rev Med Pharmacol Sci. 2018; 22: 7653-9.

58. Li D, Ji H, Niu X, Yin L, Wang Y, Gu Y, et al. Tumor-associated macrophages secrete CC-chemokine ligand 2 and induce tamoxifen resistance by activating PI3K/Akt/mTOR in breast cancer. Cancer Sci. 2020; 111: 47-58.

59. Bian $B, Y u X$, Wang $Q$, Teng T, Nie J. Atorvastatin protects myocardium against ischemia-reperfusion arrhythmia by increasing Connexin 43 expression: A rat model. European journal of pharmacology. 2015; 768: 13-20.

60. Tacheau C, Fontaine J, Loy J, Mauviel A, Verrecchia F. TGF-beta induces connexin43 gene expression in normal murine mammary gland epithelial cells via activation of p38 and PI3K/AKT signaling pathways. J Cell Physiol. 2008; 217: 759-68.

61. Leithe E, Mesnil M, Aasen T. The connexin 43 C-terminus: A tail of many tales. Bba-Biomembranes. 2018; 1860: 48-64.

62. Vivanco I, Sawyers CL. The phosphatidylinositol 3-Kinase AKT pathway in human cancer. Nat Rev Cancer. 2002; 2: 489-501.

63. Guo Q, Lu L, Liao Y, Wang X, Zhang Y, Liu Y, et al. Influence of c-Src on hypoxic resistance to paclitaxel in human ovarian cancer cells and reversal of FV-429. Cell Death Dis. 2018; 8: e3178.

64. Anbalagan M, Sheng M, Fleischer B, Zhang Y, Gao Y, Hoang V, et al. Dual Src Kinase/Pretubulin Inhibitor KX-01, Sensitizes ERalpha-negative Breast Cancers to Tamoxifen through ERalpha Reexpression. Mol Cancer Res. 2017; 15: 1491-502.

65. Gangoso E, Thirant C, Chneiweiss H, Medina JM, Tabernero A. A cell-penetrating peptide based on the interaction between c-Src and connexin43 reverses glioma stem cell phenotype. Cell death \& disease. 2014; 5. 